\title{
HOMOLOGY THEORIES FOR MULTIPLICATIVE SYSTEMS
}

\author{
BY \\ SAMUEL EILENBERG AND SAUNDERS MACLANE(1)
}

1. Introduction. The homology and cohomology groups $[1]\left({ }^{2}\right)$ of a group $\Pi$ are usually defined as the homology and cohomology groups of a certain abstract cell complex $A^{0}(\Pi)$. The $q$-dimensional cells $(q \geqq 1)$ of $A^{0}(\Pi)$ are all $q$-tuples $\left[x_{1}, \cdots, x_{q}\right]$ of elements $x_{i}$ of $\Pi$, and the boundary of such a cell is defined as

$$
\begin{aligned}
\partial\left[x_{1}, \cdots, x_{q}\right]= & {\left[x_{2}, \cdots, x_{q}\right] } \\
& +\sum_{i=1}^{q-1}(-1)^{i}\left[x_{1}, \cdots, x_{i} x_{i+1}, \cdots, x_{q}\right] \\
& +(-1)^{q}\left[x_{1}, \cdots, x_{q-1}\right],
\end{aligned}
$$

with $\partial[x]=0$. It is convenient to augment $A^{0}(\Pi)$ by regarding the commutator quotient group $\Pi /[\Pi, \Pi]$ as the group of 0 -dimensional chains, with $\partial[x]=x[\Pi, \Pi]$.

The complex $A^{0}(\Pi)$ occurs in a topological problem in which II plays the role of the fundamental group of a space. An analogous problem in which the fundamental group is replaced by a higher homotopy group has led us to believe that there exists for abelian groups a specific homology theory distinct from the one described above for all groups. This in turn suggests the thesis that the class of algebraic systems defined by any set of identities has its appropriate homology theory. The objective of this paper is a study of this thesis for the case of systems with a single operation of multiplication.

The proof that $\partial \partial=0$ in the complex $A^{0}(\Pi)$ uses only the associative law in $\Pi$, as for instance in $\partial \partial[x, y, z]=[(x y) z]-[x(y z)]$. For any associative multiplicative system $M$ one may thus define a complex $A^{0}(M)$ by the same formulas, and refer to $A^{0}$ as a "construction" on such systems $M$. A deeper property of the construction $A^{0}$ is the fact that all "formal" cycles are "formal" boundaries. These concepts are explained in more detail later in this introduction; for the moment a formal cycle may be regarded as a chain $z$ written with indeterminates as entries and such that $\partial z=0$ holds in virtue of the boundary formula (1.1) and the associative law alone.

It is important to notice that this property of $A^{0}$ is lost as soon as the commutative law is assumed for the system $M$. Indeed, the formal 2-dimen-

Received by the editors, January 9, 1951.

(1) Essential portions of the results of this paper were obtained during the tenure of a John Simon Guggenheim Fellowship by one of the authors.

(2) Numbers in brackets refer to the bibliography at the end of the paper. 
sional chain

$$
Z=[x, y]-[y, x]
$$

has boundary $\partial Z=[y x]-[x y]$, and therefore is a formal cycle as soon as the commutative law is present, but it is not a formal boundary. Thus the construction $A^{0}$ which was appropriate for the class of associative systems is no longer so for the class of associative and commutative systems. To reestablish the property that formal cycles are formal boundaries one may begin by adjoining to the construction $A^{0}$ a new 3-dimensional cell $[x \mid y]$ with

$$
\partial[x \mid y]=[x, y]-[y, x] .
$$

Note that this three-dimensional cell has only two entries, which are separated by a bar to distinguish the cell from the 2-dimensional cell $[x, y]$ already present in $A^{0}$.

One can now prove that in dimension 2 all formal cycles are formal boundaries. However, in dimension 3 we find the following formal cycles

$$
\begin{aligned}
& Z_{1}=-[x \mid y]-[y \mid x], \\
& Z_{2}=[x \mid z]-[x \mid y z]+[x \mid y]+[x, y, z]-[y, x, z]+[y, z, x], \\
& Z_{3}=[y \mid z]-[x y \mid z]+[x \mid z]-[x, y, z]+[x, z, y]-[z, x, y],
\end{aligned}
$$

which are not formal boundaries, but which we convert into formal boundaries by adjoining three new types of 4-dimensional cells:

$$
\begin{aligned}
{[x \| y] } & \text { with } & \partial[x \| y] & =Z_{1}, \\
{[x \mid y, z] } & \text { with } & \partial[x \mid y, z] & =Z_{2}, \\
{[x, y \mid z] } & \text { with } & \partial[x, y \mid z] & =Z_{3} .
\end{aligned}
$$

These additions restore the desired property in dimension 3 , and the process here started may be continued in the fashion described in $[3, \mathrm{II}]$ to yield a construction $\boldsymbol{A}$ for associative and commutative systems satisfying the condition that all formal cycles are formal boundaries in every dimension.

The constructions $A^{0}$ and $A$ are two instances of a general notion of a construction $K$ on a class $\mathcal{X}$ of multiplicative systems. Such a construction assigns to each system $M \in \mathcal{X}$ a cell complex $K(M)$. In each dimension the cells of $K(M)$ fall into several types. The cells of each type are built by arranging arbitrary elements of $M$ in an array of specified form, with a boundary formula written with indeterminates. Such a construction is called formally acyclic if every formal cycle is a formal boundary. The main theorem asserts that formally acyclic constructions exist and that any two such constructions on the same class of system $\mathcal{X}$ yield isomorphic homology theories.

The requirement of formal acyclicity is in effect a prescription for the way in which a construction can be built up one dimension at a time. Given the cells on dimension $q$ and below, each type of $(q+1)$-cell must have a 
boundary which is a formal cycle, and the requirement states that there must be enough such types of $(q+1)$-cells to convert all formal $q$-cycles into boundaries. In this sense the construction must be "maximal." This method will be used to prove the existence of formally acyclic constructions.

The notion of "formal" cycle in a construction $K$ must be made more precise. For the case of groups, we interpret formal cycles as certain cycles of the complex $K(F)$ formed for a free group $F=F_{G}$, or for a free abelian group $F=F_{G A}$. Here two distinct points of view are possible. On the one hand, one may consider every cycle in $K(F)$ as a formal cycle. Then the condition that formal cycles bound may be recorded as

$$
H_{q}(K(F))=0,
$$$$
q>1
$$

we then say that the construction $K$ is freely acyclic.

On the other hand, one may wish to have a much narrower definition of a formal cycle. Observe that in the boundary formula (1.1) for $A^{0}$ each letter $x_{i}$ appears at most once in any cell on the right, and that the same property holds for the boundary formulas used above for the enlarged construction $A$. To formalize this property, call a cell of $K(F)$ generic if its entries are products of generators of $F$, with each generator appearing at most once in the cell. Then require that the generic cells form a subcomplex $K(F, *)$ of $K(F)$. We now interpret formal cycles as cycles of this subcomplex $K(F, *)$. Thus the condition that formal cycles bound may be recorded as

$$
H_{q}(K(F, *))=0,
$$

We then say that the construction $K$ is generically acyclic.

The construction $A^{0}$ for all groups is both freely and generically acyclic, the free acyclicity being merely a restatement of the known fact that the higher homology groups of a free group are trivial. Hence, in this case both concepts of a formal cycle yield the same homology theory for groups. This is no longer the case for abelian groups. The construction $A$ outlined above will be proved in a subsequent paper to be generically acyclic. However, $A$ is not freely acyclic, since the 3-dimensional cycle $[x \mid x]$, where $x$ is a generator of the free abelian group $F$, does not bound. Thus in the case of abelian groups we have two distinct intrinsic homology theories, one based on free and the other upon generic acyclicity.

For abelian groups there are a large number of apparently quite different constructions which are generically acyclic. One such is a cubical construction $Q(\Pi)$ which is generically acyclic ( $\$ 12)$ only modulo a suitable normalization subcomplex. The main theorem for the comparison of two generically acyclic constructions also holds (\$9) when the generic acyclicity is given modulo an appropriate type of normalization. Hence the construction $Q$ and the construction $A$ partially described above have isomorphic homology theories, and the identification of these theories without explicit construction of the 
comparison is an illustration of the force of the main theorem. Exactly this comparison was requisite [3] for the topological problem mentioned above, and it was the starting point of the present investigation.

2. Free multiplicative systems. We consider multiplicative systems $M$ which have a binary operation $m_{1} m_{2} \in M$, defined for all pairs $m_{1}, m_{2} \in M$, and a unit element 1 , such that $1 m=m=m 1$ for all $m \in M$. The same symbol 1 will be used to denote the unit element in various systems $M$. If $M$ and $N$ are such systems and $\alpha$ is a function defined on $M$ and with values in $N$, then $\alpha$ is called a homomorphism (notation, $\alpha: M \rightarrow N$ ) provided

$$
\alpha(1)=1, \quad \alpha\left(m_{1} m_{2}\right)=\alpha\left(m_{1}\right) \alpha\left(m_{2}\right), \quad m_{1}, m_{2} \in M .
$$

The category [2] of all these multiplicative systems and homomorphisms will be denoted by $\mathcal{X}$.

For our purposes, a free system $F$ is a multiplicative system $F$ together with a fixed sequence of elements $g_{1}, g_{2}, \cdots$ in $F$, with $g_{1} \neq 1$, such that for any sequence $a_{1}, a_{2}, \cdots$ of elements of $F$ there exists one and only one homomorphism $\alpha: F \rightarrow F$ with $\alpha\left(g_{i}\right)=a_{i}, i=1,2, \cdots$. A multiplicative system $M$ is said to belong to $F$ if, for any sequence $m_{1}, m_{2}, \cdots$ of elements of $M$, there is one and only one homomorphism $\beta: F \rightarrow M$ with $\beta\left(g_{i}\right)=m_{i}$ for all $i$. We denote by $\mathcal{H}(F)$ the category of all multiplicative systems belonging to $F$, together with all homomorphisms $\alpha: M \rightarrow N$ of one such system into another. By definition, $F \in \mathcal{X}(F)$. One may also show that if $\alpha: M \rightarrow N$, where $M$ and $N$ belong to $F$, then the image $\alpha(M)$ and the kernel $\alpha^{-1}(1)$ of $\alpha$ belong to $F$, but we shall not use these facts. If two free systems $F$ and $F^{\prime}$ have $\mathcal{H}(F)$ $=\mathscr{H}\left(F^{\prime}\right)$, then $F$ and $F^{\prime}$ are isomorphic.

The ordinary free group $F_{G}$ with a denumerable set of generators $g_{1}, g_{2}, \cdots$ is a free system in this sense, and any group belongs to $F_{G}$. If $M$ is any multiplicative system which belongs to $F_{G}$, then $M$ is associative, and for each element $x$ in $M$ the unique homomorphism $\alpha: F_{G} \rightarrow M$ with $\alpha\left(g_{1}\right)=x$, and $\alpha\left(g_{i}\right)=1$, for $i>1$, must map $g_{1}^{-1}$ into an inverse of $x$ in $M$. Hence $M$ is a group, and the category $\mathcal{X}\left(F_{G}\right)$ of all systems belonging to the free group $F_{G}$ is exactly the category $\mathcal{X}_{G}$ of all groups. By a similar argument, the free abelian group $F_{G A}$ with a denumerable set of generators is a free system in our sense, and determines the category $\mathcal{H}\left(F_{G A}\right)$ of all abelian groups.

The universal free system $F_{I}$ with generators $g_{1}, g_{2}, \ldots$ has as its elements the "words" in these generators. A word of length 0 is the unit element 1 ; a word of length 1 is a generator $g_{i}$. Assume by induction that words of length less than $n(n>1)$ have been defined. If $w$ and $v$ are two such words of length $k, m$ respectively, with $1 \leqq k, 1 \leqq m, k+m=n$, the ordered pair [w, $v]$ is a word of length $n$. Having defined the words, we define the products in $F_{I}$ as follows: $w v=[w, v]$ if $w$ and $v$ both have positive length; $1 w=w=w 1$. Once this is done the pair notation $[w, v]$ may be abandoned since every element 
of $F$ of positive length can be written uniquely as a product of the generators, with suitable parentheses. This system $F_{I}$ is a free system, and every multiplicative system belongs to it.

One may obtain quotient systems of $F_{I}$ by introducing a reflexive, symmetric, and transitive congruence relation $w \equiv v$ on the words of $F_{I}$, provided $w \equiv v$ implies both $u w \equiv u v$ and $w u \equiv v u$. By this method we obtain additional free systems.

The free associative system $F_{A}$ is obtained by the congruence relation $w \equiv_{A} v$ which holds whenever the generators appearing in $w$ in order are identical with the generators appearing in $v$ in order. The system $F_{A}$ satisfies the associative law, and the systems belonging to it are all associative multiplicative systems.

The free associative and commutative system $F_{A C}$ is defined by the congruence relation $w \equiv_{A C} v$ which holds whenever for each $i$ the number of occurrences of $g_{i}$ in $w$ is equal to the number of occurrences of $g_{i}$ in $v$. The systems belonging to $F_{A C}$ are the systems satisfying the associative and commutative laws.

The free commutative system $F_{C}$ is defined by a congruence relation $w \equiv c^{v}$ which holds only when $w$ and $v$ have the same length, and which is defined by induction on this length as follows: $1 \equiv_{C} 1, g_{i} \equiv_{C} g_{i}$, and $w_{1} w_{2} \equiv{ }_{C} v_{1} v_{2}$, if and only if either $w_{1} \equiv c_{C} v_{1}$ and $w_{2} \equiv{ }_{C} v_{2}$, or $w_{1} \equiv c_{C} v_{2}$ and $w_{2} \equiv c_{c} v_{1}$. By induction, $F_{C}$ satisfies the commutative law. If $M$ is any commutative multiplicative system, with elements $m_{1}, m_{2}, \cdots$, there is a unique homomorphism $\alpha: F_{I} \rightarrow M$ with $\alpha g_{i}=m_{i}, i=1,2, \cdots$. Since $M$ is commutative, an easy induction shows that $w \equiv C_{C} v$ implies $\alpha w=\alpha v$ in $M$. Hence $\alpha$ induces a (unique) homomorphism of $F_{C}$ into $M$, with $\alpha g_{i}=m_{i}$. Therefore the systems belonging to $F_{C}$ are the commutative systems.

We have thus the following free systems, each with its corresponding category:

$F=F_{G}$, the free group,

$F=F_{G A}$, the free abelian group,

$F=F_{I}$, the universal system,

$F=F_{A}$, the associative system,

$F=F_{A C}$, the associative and commutative system

$F=F_{C}$, the commutative system

$$
\begin{aligned}
\mathscr{X}_{G} & =\text { all groups } \\
\mathscr{X}_{G A} & =\text { all abelian groups, } \\
\mathscr{X}_{I} & =\text { all multiplicative systems } M, \\
\mathscr{X}_{A}= & =\text { all associative } M \text { 's } \\
\mathscr{X}_{A C}= & \text { all associative and commuta- } \\
& \text { tive } M \text { 's } \\
\mathscr{X}_{C}= & \text { all commutative } M \text { 's. }
\end{aligned}
$$

More generally, one may construct from $F_{I}$ the free system satisfying any specified set of identities. An identity may be viewed as an ordered pair $\left(w_{1}, w_{2}\right)$ of words of $F_{I}$. A system $M$ is said to satisfy the identity $\left(w_{1}, w_{2}\right)$ if for every $\alpha: F_{I} \rightarrow M$, we have $\alpha w_{1}=\alpha w_{2}$. A relation $R$ on $F_{I}$ is a set of identities; that is, a set of ordered pairs. A relation $R$ is said to be closed if it is reflexive, symmetric, and transitive, and if 
(2.1) $\left(w_{1}, w_{2}\right) \in R$ implies $\left(\alpha w_{1}, \alpha w_{2}\right) \in R$ for every endomorphism $\alpha: F_{I}$ $\rightarrow F_{I}$,

(2.2) $\left(w_{1}, w_{2}\right) \in R$ implies $\left(u w_{1}, u w_{2}\right) \in R$ and $\left(w_{1} u, w_{2} u\right) \in R$ for all $u \in F_{I}$. Every relation $R$ determines its closure $\bar{R}$ as the intersection of all closed sets of identities containing $R$. Clearly $\bar{R}$ is closed. Since the set of all identities satisfied by a multiplicative system $M$ is closed, it follows that when $M$ satisfies all the identities of $R$, it satisfies all the identities of $\bar{R}$.

For any relation $R$ introduce as a new equality in the free system $F_{I}$ the congruence relation $w_{1} \equiv_{R} w_{2}$ which holds if and only if $\left(w_{1}, w_{2}\right) \in \bar{R}$. The resulting system $F_{R}$ clearly satisfies all the identities of $R$ (and hence of $\bar{R}$ ). If $M$ is any multiplicative system satisfying all the identities of $R$, and if $m_{1}, m_{2}, \cdots$ is any sequence of elements of $M$, there is a homomorphism $\alpha: F_{I} \rightarrow M$ with $\alpha g_{i}=m_{i}$. The set $S$ of all pairs $\left(w_{1}, w_{2}\right)$ of words such that $\alpha w_{1}=\alpha w_{2}$ is closed and contains $R$. Thus $\alpha$ induces a homomorphism $\alpha_{R}: F_{R}$ $\rightarrow M$, with $\alpha_{R} g_{i}=m_{i}$. Since every element of $F_{R}$ is a product of the $g_{i}$ 's, the homomorphism is unique. Hence $F_{R}$ is a free system, and the multiplicative systems belonging to it are all systems $M$ satisfying the identities of $R$, provided only that $g_{1} \neq 1$ in $F_{R}$. But if $g_{1}=1$ in $F_{R}$, then $\left(g_{1}, 1\right) \in \bar{R}$, and it follows readily by 2.1 and the transitivity of $\bar{R}$ that every identity $\left(w_{1}, w_{2}\right)$ belongs to $\bar{R}$. We have proved the following theorem.

THEOREM 2.1. Let $R$ be any set of identities such that its closure is not the set of all identities. Then there exists one and, up to isomorphism, only one, free system $F_{R}$ such that the multiplicative systems belonging to $F_{R}$ are exactly the systems satisfying the identities $R$.

In particular, we may consider the four standard examples of relations:

$I$ the empty set of relations,

$C$ the set containing only the "commutative" relation $\left(g_{1} g_{2}, g_{2} g_{1}\right)$,

$A$ the set containing the "associative" relation $\left(g_{1}\left(g_{2} g_{3}\right),\left(g_{1} g_{2}\right) g_{3}\right)$,

$A C$ the set $A \cup C$.

In the case $R=C$ the free commutative system $F_{C}$ explicitly constructed above is such that the multiplicative systems belonging to it are exactly the systems satisfying the commutative law $g_{1} g_{2}=g_{2} g_{1}$, and thus $F_{C}$ satisfies the conditions of the theorem for $R=C$, and is in fact identical with the system $F_{R}$ constructed by the method of Theorem 2.1. The explicit description of $F_{C}$ above is a description of the closure of the relation $C$ as the set of all $(w, v)$ with $w \equiv c v$. The same remark applies in the other cases of $R$ listed above.

Our definition of a free system $F$ does not imply that every element of $F$ is a product of generators; indeed, this is not the case for the free group $F_{G}$, because of the presence of inverses of generators. However, $F_{G}, F_{G A}$, and all the free systems $F_{R}$ satisfy the following condition.

Finiteness Condition. For each $x \in F$ there exists an integer $n$ such that 
$\theta_{n} x=x$ holds for the projection $\theta_{n}: F \rightarrow F$ with

$$
\theta_{n} g_{i}=g_{i}, \quad i=1, \cdots, n ; \quad \theta_{n} g_{j}=1, \quad j=n+1, n+2, \cdots .
$$

Subsequently we assume that all free systems considered satisfy this finiteness condition. From it we deduce the following lemma.

Lемма 2.2. If $x_{1}, \cdots, x_{k}$ is a finite list of elements of the free system $F$, there exists an integer $n$ such that the projection $\theta_{n}$ defined by (2.3) has $\theta_{n} x_{1}$ $=x_{1}, \cdots, \theta_{n} x_{k}=x_{k}$.

Proof. By the finiteness condition choose such an integer $n_{j}$ for each $x_{j}$. Then any $n$ larger than $n_{1}, \cdots, n_{k}$ has the required property.

3. Generic endomorphisms. In the universal free system $F_{I}$ with generators $g_{1}, g_{2}, \cdots$ a word $w$ is called generic if no generator is repeated in the representation of $w$ as a product of the $g$ 's. If $F$ is any free system relative to the sequence $g_{1}^{\prime}, g_{2}^{\prime}, \cdots$, there is a unique homomorphism $\mu: F_{I} \rightarrow F$ with $\mu g_{i}=g_{i}{ }^{\prime}$. We say that an element $x$ of $F$ is represented by the word $w$ of $F_{I}$ if $\mu w=x$. Note that not every element of $F$ need have a representation.

LEMMA 3.1. If two generic words $w_{1}$ and $w_{2}$ of $F_{I}$ represent the same element $x$ of the free system $F$, then $w_{1}$ and $w_{2}$ involve the same generators.

Proof. Suppose to the contrary that $g_{i}$ is a generator of $F_{I}$ which appears in $w_{1}$ but not in $w_{2}$. Choose $\beta: F \rightarrow F$, so that $\beta\left(g_{i}^{\prime}\right)=g_{1}^{\prime}, \beta\left(g_{j}^{\prime}\right)=1$ for $j \neq i$. Then $\beta \mu: F_{I} \rightarrow F$. Since $g_{i}$ occurs only once in $w_{1}$ we have $\beta \mu\left(w_{1}\right)=g_{1}^{\prime}$. Since $g_{i}$ does not occur in $w_{2}$, we have $\beta \mu\left(w_{2}\right)=1$. Thus $g_{1}^{\prime}=1$, contrary to the first condition in the definition of a free system.

An element $x$ in the free system $F$ is generic if it can be represented by a generic word. In view of Lemma 3.1 we can speak of the generators involved in the generic element $x \in F$. The generic elements $x_{1}, \cdots, x_{n}, \cdots$ of $F$ are mutually disjoint if no two of them involve the same generator. The element 1 is disjoint with any generic element.

An endomorphism $\alpha: F \rightarrow F$ is called generic if $\alpha$ carries disjoint generic elements into disjoint generic elements. A composite of generic endomorphisms is again generic. It is also easy to see that an endomorphism $\alpha F \rightarrow F$ is generic if and only if the images $\alpha\left(g_{i}\right)$ of all the generators are generic and mutually disjoint.

Let $\Phi^{*}$ denote the set of all generic endomorphisms of $F$. A set $\Phi$ of endomorphisms of $F$ is termed admissible if $\Phi$ is closed under composition and contains $\Phi^{*}$. A sequence $x_{1}, \cdots, x_{n}$ of elements of $F$ is called a $\Phi$-sequence if the unique endomorphism $\alpha: F \rightarrow F$ with $\alpha\left(g_{i}\right)=x_{i}$ for $i=1, \cdots, n$, and $\alpha\left(g_{j}\right)=1$ for $j>n$, is in $\Phi$. In particular $(n=1)$ the element $x$ is a $\Phi$-element of $F$ if $\alpha$ with $\alpha\left(g_{1}\right)=x, \alpha\left(g_{j}\right)=1$, for $j>1$, is in $\Phi$.

The set $\Phi^{*}$ of generic endomorphisms of $F$ is admissible; the $\Phi^{*}$-elements are the generic elements of $F$, and the $\Phi^{*}$-sequences $x_{1}, \cdots, x_{n}$ of $F$ are 
exactly the sequences of disjoint generic elements of $F$. The set $\Phi^{0}$ of all endomorphisms is also admissible, and any sequence of elements of $F$ is a $\Phi^{0}$-sequence. In order to state simultaneously theorems about free acyclicity and generic acyclicity in the sense described in the introduction, we shall state below theorems relative to any admissible set $\Phi$ of endomorphisms, with the cases $\Phi=\Phi^{0}$ (free acyclicity) and $\Phi=\Phi^{*}$ (generic acyclicity) included.

For the free groups $F_{G}$ and $F_{G A}$ one may also introduce pseudo-generic elements as those products of generators, and their inverses, in which each generator $g$ appears at most once (as $g$ or as $g^{-1}$ ). A pseudo-generic endomorphism $\alpha$ is then an endomorphism carrying the generators into disjoint pseudo-generic elements, and the set $\Phi^{\#}$ of pseudo-generic endomorphisms is admissible.

LEMMA 3.2. If $\Phi$ is any admissible set of endomorphisms of the free system $F$, and $\xi=\left(x_{1}, \cdots, x_{n}\right)$ any $\Phi$-sequence of elements of $F$, then

(i) any permutation of $\xi$ is a $\Phi$-sequence,

(ii) any subsequence of $\xi$ is a $\Phi$-sequence,

(iii) the sequence $\left(x_{1} x_{2}, x_{3}, \cdots, x_{n}\right)$ is a $\Phi$-sequence.

Proof. Conclusion (i) is immediate, since any permutation of $g_{1}, \cdots, g_{n}$ can be achieved by a generic endomorphism $\alpha$, where $\alpha \in \Phi$; and (ii) is established in similar fashion. To obtain (iii), observe that the endomorphism $\beta: F \rightarrow F$ with $\beta\left(g_{1}\right)=g_{1} g_{2}, \beta\left(g_{i}\right)=g_{i+1}$ for $i>1$ is a generic endomorphism and hence in $\Phi$. Thus, if the unique endomorphism $\alpha$ with $\alpha\left(g_{i}\right)=x_{i}, i=1, \cdots, n$, and $\alpha\left(g_{j}\right)=1, j>n$, lies in $\Phi$, the composite $\beta \alpha$ is in $\Phi$, and $(\beta \alpha) g_{i}=x_{1} x_{2}$, $(\beta \alpha)\left(g_{i}\right)=x_{i-1}$, for $i=2, \cdots, n$, so that $\left(x_{1} x_{2}, x_{3}, \cdots, x_{n}\right)$ is a $\Phi$-sequence by definition.

To each multiplicative system $M$ we assign an abelian group $H(M)$, as follows. Let $C(M)$ be the free abelian group with generators $\{x\}$, one for each $x \in M$, and $B(M)$ the subgroup of $C(M)$ spanned by all elements $\{y\}-\{x y\}+\{x\}$, for $x, y \in M$. Then define $H(M)$ as $C(M) / B(M)$. This definition insures that the mapping $\mu_{M}: M \rightarrow H(M)$ defined by $\mu_{M}(x)$ $=\{x\}+B(M)$ is a homomorphism

$$
\mu_{M}: M \rightarrow H(M) .
$$

It is easy to see that any homomorphism $\gamma: M \rightarrow G$ of $M$ into an abelian group $G$ admits a unique factorization $\gamma=\beta \mu_{M}$, where $\beta: H(M) \rightarrow G$; this property characterizes $H(M)$ and $\mu_{M}$ up to an isomorphism. If $M$ is a group, $H(M)$ is therefore the factor commutator group $M /[M, M]$, and $\mu_{M}$ is the mapping $\mu_{M}(m)=m[M, M]$.

Each homomorphism $\alpha: M \rightarrow N$ induces a homomorphism $H(\alpha): H(M)$ $\rightarrow H(N)$ with $\mu_{N} \alpha=H(\alpha) \mu_{M}$ and $H\left(\alpha \alpha^{\prime}\right)=H(\alpha) H\left(\alpha^{\prime}\right)$, whenever $\alpha \alpha^{\prime}$ is defined.

Let $\Phi$ be an admissible set of endomorphisms of a free system $F$, and denote by $H(F, \Phi)$ the subgroup of $H(F)$ spanned by the elements $\mu_{F} x$ for $x$ a 
$\Phi$-element of $F$. The elements of $H(F, \Phi)$ will be called the $\Phi$-elements of $H(F)$. If $\alpha \in \Phi$, then $H(\alpha)$ maps $H(F, \Phi)$ into $H(F, \Phi)$; thus $H(\alpha)$ defines operators of the system $\Phi$ on the abelian group $H(F, \Phi)$.

4. Complexes. A sequence of abelian groups and homomorphisms

$$
\cdots \leftarrow C_{q-1} \stackrel{\partial_{q}}{\longleftarrow} C_{q} \stackrel{\partial_{q+1}}{\longleftarrow} C_{q+1} \leftarrow \cdots
$$

$(q=0, \pm 1, \pm 2, \cdots)$ such that each composition $\partial_{q} \partial_{q+1}: C_{q+1} \rightarrow C_{q-1}$ is the zero homomorphism is called a chain complex. If $K$ denotes the chain complex, we shall write $C_{q}(K)$ for $C_{q}$. The dimension index $q$ in the boundary operator $\partial_{q}$ will usually be omitted.

A map $F: K \rightarrow L$ of a chain complex $K$ into a chain complex $L$ is a sequence of homomorphisms $f_{q}: C_{q}(K) \rightarrow C_{q}(L)$ such that $\partial_{q} f_{q}=f_{q-1} \partial_{q}$. A homotopy $D: f^{0} \simeq f^{1}$ of two maps $f^{0}, f^{1}: K \rightarrow L$ is a sequence of homomorphisms $D_{q}: C_{q}(K) \rightarrow C_{q+1}(L)$ such that $\partial_{q+1} D_{q}+D_{q-1} \partial_{q}=f_{q}^{1}-f_{q}^{0}$. We shall of ten speak of maps or homotopies defined only in dimensions $q \leqq n$.

A chain complex $K$ is called an (abstract) cell complex, provided that $C_{q}(K)=0$ for $q<0$, and provided that in each group $C_{q}(K)$ a set $\left\{\sigma^{q}\right\}$ is given, which freely generates the group $C_{q}(K)$. The generators $\sigma^{q}$ are called $q$-cells of $K$.

A cell complex is also a chain complex, and the concepts of "map" and "homotopy" are defined as before.

One has to be cautious about the concept of "subcomplex." A chain complex $L$ is said to be a subcomplex of the chain complex $K$ if each $C_{q}(L)$ is a subgroup of $C_{q}(K)$ and $\partial_{q}$ in $L$ agrees with $\partial_{q}$ in $K$. If $K$ and $L$ are cell complexes we require further that each cell of $L$ also be a cell of $K$. It is understood that the "empty" subcomplex consists of the zero subgroup in each dimension.

The usual definition of the homology and cohomology groups (over an abelian coefficient group $G$ ) of a chain complex $K$ modulo a subcomplex $L$ will not be repeated here. We shall deal chiefly with homology groups with integer coefficients. These will be denoted by $H_{q}(K, L)$ or by $H_{q}(K)$ if $L=0$.

A map $f: K \rightarrow L$ induces homomorphisms $f_{*}: H_{q}(K ; G) \rightarrow H_{q}(L ; G)$ and $f^{*}: H^{q}(L ; G) \rightarrow H^{q}(K ; G)$ of the homology and cohomology groups. If $f^{0}$ and $f^{1}$ are homotopic, then $f_{*}^{0}=f_{*}^{1}$ and $f^{0 *}=f^{1 *}$.

5. Constructions. Throughout this section, $F$ is a fixed free multiplicative system and $\Phi$ a fixed admissible set of endomorphisms of $F$. A $\Phi$-construction $K$ on $\mathscr{X}(F)$ is a function which assigns to each system $M$ in $\mathscr{X}(F)$ an abstract cell complex $K(M)$ satisfying the following conditions:

(5.0) $K(M)$ has no cells of dimension less than 1.

(5.1) The $q$-cells of $K(M)$ fall into types. The division into types is independent of $M$.

(5.2) To each type $t$ of $q$-cells there corresponds an integer $r=r(t) \geqq 0$ 
called the number of entries for the type in question. The $q$-cells $\sigma$ of type $t$ in any $K(M)$ are the $r$-tuples $\left[x_{1}, \cdots, x_{r}\right]_{t}$, where $x_{1}, \cdots, x_{r} \in M$.

(5.3) If $\alpha: M_{1} \rightarrow M_{2}$, then the definition $K(\alpha)\left[x_{1}, \cdots, x_{r}\right]_{t}=\left[\alpha x_{1}, \cdots\right.$, $\left.\alpha x_{r}\right]_{t}$ yields a map $K(\alpha): K\left(M_{1}\right) \rightarrow K\left(M_{2}\right)$; that is, $K(\alpha) \partial=\partial K(\alpha)$.

(5.4) The $\Phi$-cells of $K(F)$; that is, all the cells $\left[x_{1}, \cdots, x_{r}\right]_{t}$ of $K(F)$ with $x_{1}, \cdots, x_{r}$ a $\Phi$-sequence, constitute a subcomplex $K(F, \Phi) \subset K(F)$.

The "type" of a cell will normally be indicated by some method other than the affixing of a subscript $t$, as illustrated by the case of the cells $[x, y, z]$ and $[x \mid y]$ discussed in the introduction.

It follows from (5.2) that if for some type $t$ we have $r(t)=0$, that is, if the number of entries for this type is zero, then there is only one cell [ ] $]_{t}$ of type $t$, and this cell is common to all the complexes $K(M)$ and the complex $K(F, \Phi)$.

Condition (5.3) implies that if $S$ is a subsystem of $M$ and $\kappa: S \rightarrow M$ is the inclusion map, then $K(\kappa)$ maps $K(S)$ isomorphically onto a subcomplex of $K(M)$, and therefore we may regard $K(S)$ as a subcomplex of $K(M)$.

Condition (5.3) states in effect that the boundary of a cell of any type $t$ is given by one "formula" valid for all $M$. Indeed, consider the "typical" cell $\tau=\left[g_{1}, \cdots, g_{r}\right]_{t}$ of type $t$ in $K(F)$ and its boundary

$$
\partial \tau=a_{1} \tau_{1}+\cdots+a_{s} \tau_{s}
$$

where the $a_{i}$ are nonzero integers and the $\tau_{i}$ distinct cells of dimension one lower than $\tau$. The admissible projection $\theta_{r}: F \rightarrow F$ with $\theta_{r} g_{i}=g_{i}, i=1, \cdots, r$, and $\theta_{r} g_{j}=1$ otherwise has $K\left(\theta_{r}\right) \tau=\tau$, and hence by (5.4), $K\left(\theta_{r}\right) \partial \tau=\partial \tau$. Then if $\tau_{i}^{\prime}=K\left(\theta_{r}\right) \tau_{i}$, one has

$$
\partial \tau=a_{1} \tau_{1}^{\prime}+\cdots+a_{8} \tau_{8}^{\prime},
$$

with $K\left(\theta_{r}\right) \tau_{i}^{\prime}=\tau_{i}^{\prime}$ for each $i$. If therefore in the system $F$ every element is a product of generators, it follows that each entry of each $\tau_{i}^{\prime}$ is a product of certain of the generators $g_{1}, \cdots, g_{r}$, with possible repetitions. If we replace the generators $g_{1}, \cdots, g_{r}$ by indeterminates $x_{1}, \cdots, x_{r}$, we obtain a boundary "formula," which by (5.3) is valid for all cells of type $t$. A similar result applies when elements of $F$ can be written as products of generators and their inverses.

The most important case considered later is that when $\Phi=\Phi^{*}$ is the set of all generic endomorphisms. We then speak of a generic construction, and call the cells and chains of $K\left(F, \Phi^{*}\right)=K(F, *)$ generic cells and chains. In terms of the boundary formula explained above, condition (5.4) means that in the formula for $\partial\left[x_{1}, \cdots, x_{r}\right]_{t}$ no indeterminate $x_{i}$ is repeated in any one term of the boundary. A similar remark applies to pseudo-generic constructions.

An augmented $\Phi$-construction $\bar{K}$ is a $\Phi$-construction $K$ together with a set of homomorphisms 


$$
\partial_{M}^{\prime}: C_{1}(K(M)) \rightarrow H(M),
$$

where $H(M)$ is the group defined at the end of $\S 3$, subject to the conditions:

(5.5) The composite homomorphism $\partial_{M}^{\prime} \partial: C_{2}(K(M)) \rightarrow H(M)$ is zero.

(5.6) If $\alpha: M_{1} \rightarrow M_{2}$, then $\partial_{M_{2}}^{\prime} K(\alpha)=H(\alpha) \partial_{M_{1} \text {. }}^{\prime}$

(5.7) $\partial_{F}^{\prime}$ carries $C_{1}(K(F, \Phi))$ into the subgroup $H(F, \Phi)$ of $H(F)$.

In virtue of (5.5), $K(M)$ may be enlarged to a chain complex $\bar{K}(M)$ by adjoining $H(M)$ as the group of 0 -chains. By (5.7), the analogous adjunction of $H(F, \Phi)$ to $K(F, \Phi)$ yields the $\Phi$-subcomplex $\bar{K}(F, \Phi)$ of $\bar{K}(F)$. Because of (5.6) each $\alpha: M_{1} \rightarrow M_{2}$ yields a map $\bar{K}(\alpha): \bar{K}\left(M_{1}\right) \rightarrow \bar{K}\left(M_{2}\right)$.

We shall need to compare various constructions on the same category $\mathcal{H}(F)$. A map $f: K \rightarrow L$ of one such construction onto another is a family of maps $f_{M}: K(M) \rightarrow L(M)$ defined for each $M \in \mathcal{X}(F)$ and such that:

(5.8) For each $\alpha: M_{1} \rightarrow M_{2}$ one has $f_{M_{2}} K(\alpha)=L(\alpha) f_{M_{1}}$.

(5.9) The map $f_{F}$ carries $K(F, \Phi)$ onto $L(F, \Phi)$.

A homotopy $D: f^{0} \simeq f^{1}$ of two maps $f^{0}, f^{1}: K \rightarrow L$ is a family of homotopies $D_{M}: f_{M}^{0} \rightarrow f_{M}^{1}$ such that:

(5.10) For each $\alpha: M_{1} \rightarrow M_{2}$ one has $D_{M_{2}} K(\alpha)=L(\alpha) D_{M_{1}}$.

(5.11) The homotopy $D_{F}$ carries $K(F, \Phi)$ into $L(F, \Phi)$.

For maps $f: \bar{K} \rightarrow \bar{L}$ of augmented constructions we further require that

$$
\partial_{M}^{\prime} c=\partial_{M}^{\prime} f_{M} c, \text { for each } c \in G_{1}(K(M)) .
$$

This condition means that $f_{M}$ may be extended to a map $\bar{f}_{M}$ of $\bar{K}(M)$ into $\bar{L}(M)$ by choosing $\bar{f}_{M}$ as the identity in dimension 0 . Similarly, any homotopy $D: f^{0} \simeq f^{1}$ of two maps $f^{0}, f^{1}: K \rightarrow L$ extends to a homotopy $\bar{D}: \bar{f}_{M}^{0} \simeq \bar{f}_{M}^{1}$, by setting $\bar{D}_{M}=0$ in dimension 0 .

6. Acyclic constructions. An augmented $\Phi$-construction $\bar{K}$ is $\Phi$-acyclic, if

$$
H_{q}(\bar{K}(F, \Phi))=0,
$$$$
q=0,1,2, \cdots \text {. }
$$

The main comparison theorem is as follows.

TheOREM 6.1. Let $\bar{K}$ and $\bar{L}$ be two augmented $\Phi$-constructions on the same category $\mathcal{X}(F)$, with $\bar{L} \Phi$-acyclic. Then there exists a map $f: \bar{K} \rightarrow \bar{L}$, and any two such maps are homotopic. If (6.1) holds only for $q \leqq n$, then $f$ may be defined in dimensions not greater than $n+1$, and the homotopy between two such maps exists in dimensions not greater than $n$.

The proof depends upon defining $f$ and the homotopy $D$ first for typical cells. For each type $t$ of cell of $K$ with $r=r(t)$ entries, the cell $\tau=\left[g_{1}, \cdots, g_{r}\right]_{t}$ formed with the first $r$ generators of $F$ is the typical cell of type $t$. If $\sigma$ $=\left[x_{1}, \cdots, x_{r}\right]_{t} \in K(M)$ is another cell of type $t$, then $\sigma$ may be written as

$$
\sigma=K(\alpha) \tau,
$$

where $\alpha: F \rightarrow M$ is the unique homomorphism with $\alpha\left(g_{i}\right)=x_{i}$ for $i=1, \cdots, r$ 
and $\alpha\left(g_{i}\right)=1$, for $i>r$. The representation (6.2) will be called the canonical representation of $\sigma$. If $\beta: M \rightarrow M_{1}$, then it is easy to see that

$$
K(\beta) \sigma=K(\beta \alpha) \tau
$$

is the canonical representation of the cell $K(\beta) \sigma$ of $K\left(M_{1}\right)$. The cell $\tau$ itself has the canonical representation $\tau=K\left(\theta_{r}\right) \tau$, where $\theta_{r}$ is the projection $\theta_{r}: F \rightarrow F$ with $\theta_{r}\left(g_{i}\right)=g_{i}, i=1, \cdots, r$, and $\theta_{r}\left(g_{j}\right)=1, j>r$. The definition of $K(F, \Phi)$ indicates that the cell $\sigma$ of $K(F)$ lies in $K(F, \Phi)$ if and only if the map $\alpha$ of its canonical representation (6.2) lies in $\Phi$. In particular, the typical cell $\tau$ is in $K(F, \Phi)$, and any endomorphism $\beta$ of $\Phi$ maps $K(F, \Phi)$ into itself.

To define the map $f: \bar{K} \rightarrow \bar{L}$ in dimension 1 , let $\tau=\left[g_{1}, \cdots, g_{r}\right]_{t}$ be the typical cell of $K$ of type $t$ in dimension 1 . Then $\partial_{F}^{\prime} \tau \in H(F, \Phi)=C_{0}(\bar{K}(F, \Phi))$ by condition (5.7). Hence by the hypothesis (6.1) for $q=0, \partial_{F}^{\prime} \tau$ is a boundary in $\bar{L}(F, \Phi)$, so has the form $\partial_{F}^{\prime} \tau=\partial_{F}^{\prime} c(\tau)$, for some $c(\tau) \in C_{1}(\bar{L}(F, \Phi))$. For any 1-cell $\sigma$ of $K(M)$ with the canonical representation $\sigma=K(\alpha) \tau$ of (6.2) we define

$$
f_{M} \sigma=L(\alpha) c(\tau) .
$$

Then $\partial_{M}^{\prime} \sigma=\partial_{M}^{\prime} f_{M} \sigma$, as required by (5.12), for by (5.6)

$$
\begin{aligned}
\partial_{M}^{\prime} f_{M} \sigma & =\partial_{M}^{\prime} L(\alpha) c(\tau)=H(\alpha) \partial_{F}^{\prime} c(\tau)=H(\alpha) \partial_{F}^{\prime} \tau \\
& =\partial_{M}^{\prime} K(\alpha) \tau=\partial_{M}^{\prime} \sigma .
\end{aligned}
$$

If $\beta: M \rightarrow M_{1}$, then $K(\beta \alpha) \tau$ is the canonical representation of $K(\beta) \sigma$, and

$$
\begin{aligned}
f_{M_{1}} K(\beta) \sigma & =f_{M_{1}} K(\beta \alpha) \tau=L(\beta \alpha) c(\tau) \\
& =L(\beta) L(\alpha) c(\tau)=L(\beta) f_{M} \sigma .
\end{aligned}
$$

Finally, if $\sigma=K(\alpha) \tau$ is a 1-cell of $K(F, \Phi)$, then $\alpha \in \Phi, c(\tau) \in L(F, \Phi)$, hence the definition of $f$ gives $f_{F} \sigma \in L(F, \Phi)$. The map $f$ thus satisfies the requisite conditions (5.8), (5.9), and (5.12) in dimension 1.

Now proceed by induction, and suppose that a map $f: \bar{K} \rightarrow \bar{L}$ has been defined in all dimensions $q<m$, for some $m>1$. Let $\tau=\left[g_{1}, \cdots, g_{r}\right]_{t}$ be the typical cell of $K$ of type $t$ in dimension $m$. Then $\partial \tau$ is an $m-1$ chain of $K(F, \Phi)$ so that $f_{F} \partial \tau$ is defined. Furthermore $\partial f_{F} \partial \tau=f_{F} \partial \partial \tau=0$, so that $f_{F} \partial \tau$ $\in Z_{m-1}(L(F, \Phi))$. By hypothesis (6.1) for $q=m-1$ it follows that there is an $m$-chain $c(\tau)$ in $C_{m}(L(F, \Phi))$ such that

$$
\partial c(\tau)=f_{F} \partial \tau .
$$

For any cell $\sigma$ of $K(M)$ with the canonical representation $\sigma=K(\alpha) \tau$ of (6.2) we now define

$$
f_{M} \sigma=L(\alpha) c(\tau),
$$

and as above we verify that $f$ satisfies the conditions (5.8) and (5.9) for a 
map in dimension $m$.

Now let $f^{0}, f^{1}: \bar{K} \rightarrow \bar{L}$ be two maps, and assume that a homotopy $D: f^{0} \simeq f^{1}$ is already defined for all cells of dimension less than $m$, for some $m>0$.

In case $m=1$, choose any typical 1-cell $\tau$ of $K(F, \Phi)$ as above. The chain $z=f^{1} \tau-f^{0} \tau \in C_{1}(L(F, \Phi))$ is a cycle of $\bar{L}(F, \Phi)$, since by (5.12)

$$
\partial_{F}^{\prime} z=\partial_{F}^{\prime} f^{1} \tau-\partial_{F}^{\prime} f^{0} \tau=\partial_{F}^{\prime} \tau-\partial_{F}^{\prime} \tau=0 .
$$

In case $m>1$, again choose a typical cell $\tau$ of $K$ and consider the chain

$$
z=f^{1} \tau-f^{0} \tau-D \partial \tau \in C_{m}(L(F, \Phi)) .
$$

Then

$$
\begin{aligned}
\partial z & =\partial f^{1} \tau-\partial f^{0} \tau-\partial D \partial \tau \\
& =\partial f^{1} \tau-\partial f^{0} \tau-\left(f^{1} \partial \tau-f^{0} \partial \tau-D \partial \partial \tau\right)=0 .
\end{aligned}
$$

Thus $z$ is again a cycle of $L(F, \Phi)$.

In both cases $m=1$ and $m>1$ there is then by hypothesis (6.1) with $q=m$ a chain $d(\tau) \in C_{m+1}(L(F, \Phi))$ such that $\partial d(\tau)=z$. For any $m$-cell with canonical representation $\sigma=K(\alpha) \tau$ define

$$
D \sigma=L(\alpha) d(\tau) \text {. }
$$

Then it follows as before that

$$
\partial D \sigma+D \partial \sigma=f^{1} \sigma-f^{0} \sigma
$$

and, for $\beta: M \rightarrow M_{1}$, that

$$
D K(\beta) \sigma=L(\beta) D \sigma,
$$

while, as previously, $D$ carries $K(F, \Phi)$ into $L(F, \Phi)$. This completes the construction of the desired homotopy.

THEOREM 6.2. For any free multiplicative system $F$ and any admissible set $\Phi$ of endomorphisms of $F$ there exists a $\Phi$-acyclic augmented construction $\bar{K}$ on the category $\mathcal{X}(F)$, and any two such constructions $\bar{K}$ and $\bar{L}$ are chain equivalent, in the sense that there exist maps $f: \bar{K} \rightarrow \bar{L}$ and $g: \bar{L} \rightarrow \bar{K}$ and homotopies $D: g f \simeq I$, $E: f g \simeq I$, where $I$ is the appropriate identity map. In particular, $f$ and $g$ induce isomorphisms between the homology and cohomology groups of $K(M)$ and $L(M)$ for each $M$ in $\mathcal{X}(F)$. The same applies to the groups of $\bar{K}(M)$ and $\bar{L}(M)$.

In virtue of this theorem, given a category $\mathcal{X}(F)$ and an admissible class $\Phi$ of endomorphisms, we may speak of the $\Phi$-acyclic homology and cohomology groups of each system $M \in \mathcal{X}(F)$, without explicit reference to any particular construction. For instance, the integral homology groups $H_{q}(M)$ are the groups obtained from the complex $K(M)$, for any augmented $\Phi$-acyclic construction $\bar{K}$ on the category $\mathscr{H}(F)$. To determine $H_{q}(M)$ one may also 
use any augmented $\Phi$-construction $\bar{K}, \Phi$-acyclic in the sense of (6.1) in dimensions not greater than $q$; indeed, the existence proof shows that $\bar{K}$ may be made acyclic in all dimensions by adding cells of dimensions greater than $q+1$. Furthermore, if $\alpha: M_{1} \rightarrow M_{2}$ is any homomorphism between two systems $M_{1}, M_{2} \in \mathcal{H}(F)$, the induced homomorphism $\alpha_{*}: H_{q}\left(M_{1}\right) \rightarrow H_{q}\left(M_{2}\right)$ may be used without reference to its explicit definition as the homomorphism induced by the map $K(\alpha): K\left(M_{1}\right) \rightarrow K\left(M_{2}\right)$ obtained from a particular construction; for the theorem, together with the property (5.8) of the mapping $f$, shows that this induced homomorphism is independent of the choice of the $\Phi$-acyclic construction.

Proof. Only the existence remains to be established. We use the following consequence of the finiteness condition of $\$ 2$.

LeMma 6.3. For every augmented $\Phi$-construction $\bar{K}$ and every chain $c$ of $\bar{K}(F)$ there is an integer $k$ such that the projection $\theta_{k}: F \rightarrow F$ with $\theta g_{i}=g_{i}, i=1$, $\cdots, k$, and $\theta g_{j}=1$, for $j>k$, satisfies $\bar{K}\left(\theta_{k}\right) c=c$.

Suppose first that $c$ is of dimension 0 . Then by the definition of the group $H(F)$ of zero chains of $\bar{K}$ there is a set of elements $x_{1}, \cdots, x_{p}$ of $F$ such that $c$ is a linear combination of the elements $\mu_{F}\left(x_{i}\right) \in H(F)$. By Lemma 2.2 there exists an integer $k$ such that the corresponding $\theta$ satisfies $\theta x_{j}=x_{j}, j=1, \cdots, p$. Therefore $\bar{K}(\theta) \mu_{F}\left(x_{i}\right)=H(\theta) \mu_{F}\left(x_{i}\right)=\mu_{F}\left(\theta x_{i}\right)=\mu_{F}\left(x_{i}\right)$, so that $\bar{K}(\theta) c=c$, as asserted.

Next suppose that $c$ has dimension greater than 0 . Let $x_{1}, \cdots, x_{p}$ be a list of all the entries in all the cells of $c$. Then as before there is a projection $\theta$ with $\theta x_{j}=x_{j}$, for $j=1, \cdots, p$, and hence $\bar{K}(\theta) c=c$.

Existence proof. Suppose by induction on $n$ that there exists an augmented $\Phi$-construction $\bar{K}^{(n)}$ with $H_{q}\left(\bar{K}^{(n)}(F, \Phi)\right)=0$, for $0 \leqq q<n$. In case $n=0$, we may choose $\bar{K}^{(0)}$ to be the void construction with the obvious augmentation to start the induction. A set $Z \subset Z_{n}\left(\bar{K}^{(n)}(F, \Phi)\right)$ will be called a pseudo-generating set if the homology classes of the cycles $K^{(n)}(\alpha) Z$, where $z$ ranges over $Z$ and $\alpha$ ranges over $\Phi$, generate the group $H_{n}\left(\bar{K}^{(n)}(F, \Phi)\right)$. For each $z \in Z$ we choose an integer $k$ by Lemma 6.3 , so that the corresponding projection $\theta_{k}$ has $\bar{K}^{(n)}\left(\theta_{k}\right) z=z$. We introduce a new cell $\sigma=\left[x_{1}, \cdots, x_{k}\right]_{z}$ of dimension $n+1$, type $z$, and $k$ entries, with boundary

$$
\partial \sigma=\partial\left[x_{1}, \cdots, x_{k}\right]_{z}=\bar{K}^{(n)}(\alpha) z,
$$

where $\sigma=\bar{K}^{(n+1)}(\alpha)\left[g_{1}, \cdots, g_{k}\right]_{z}$ is the canonical representation of $\sigma$. The resulting $\bar{K}^{(n+1)}$ is manifestly an augmented $\Phi$-construction. The canonical representation of the cell $\sigma=\left[g_{1}, \cdots, g_{k}\right]_{z}$ has $\alpha=\theta_{k}$, hence $\partial \sigma=\bar{K}^{(n)}\left(\theta_{k}\right) z=z$. Thus $\bar{K}^{(n+1)}$ satisfies condition (6.1) for $q=0, \cdots, n$. This completes the proof of Theorem 6.2.

This argument also shows that any augmented $\Phi$-construction $\bar{K}^{(0)}$ may 
be enlarged (by adding new types of cells) to a construction $\bar{K}$ which is $\Phi$ acyclic.

For each $\Phi$-construction $K$ and each $\alpha \in \Phi$ the map $K(\alpha)$ carries $K(F, \Phi)$ into $K(F, \Phi)$ and satisfies $K\left(\alpha \alpha^{\prime}\right)=K(\alpha) K\left(\alpha^{\prime}\right)$. Thus, through $K(\alpha), \Phi$ operates on the groups of chains, cycles, and homology groups of the complex $K(F, \Phi)$. Similarly, the endomorphisms $H(\alpha)$ of $H(F, \Phi)$ defined in $\S 3$ yield operators of $\Phi$ on $H(F, \Phi)$. We therefore may speak of operator homomorphisms and operator isomorphisms between these various groups.

Theorem 6.4. A $\Phi$-construction $K$ can be augmented to a $\Phi$-acyclic construction $\bar{K}$ if and only if

$$
\begin{aligned}
& H_{1}(K(F, \Phi)) \text { is operator isomorphic to } H(F, \Phi), \\
& H_{q}(K(F, \Phi))=0,
\end{aligned}
$$$$
q=2,3, \cdots .
$$

Proof. Suppose first that $K$ is so augmentable. The homomorphism $\partial_{F}^{\prime}: C_{1}(K(F, \Phi)) \rightarrow H(F, \Phi)$ is onto $H(F, \Phi)$ because $H_{0}(\bar{K}(F, \Phi))=0$, and has as kernel the group $B_{1}(K(F, \Phi))$ of one-dimensional boundaries of $K(F, \Phi)$ because $H_{1}(\bar{K}(F, \Phi))=0$. Thus $\partial_{F}^{\prime}$ induces an operator isomorphism, as required in (6.6), and the conditions cited are thus necessary.

Conversely, assume (6.6) and (6.7). By (6.6) we may choose an operator homomorphism $\lambda: C_{1}(K(F, \Phi)) \rightarrow H(F, \Phi)$ onto $H(F, \Phi)$ with kernel $B_{1}(K(F, \Phi))$. Let $\sigma$ be any 1-cell of $K(M)$ with the canonical representation $\sigma=K(\alpha) \tau, \tau$ typical. Define the augmentation mapping $\partial_{M}^{\prime}$ as

$$
\partial_{M}^{\prime} \tau=H(\alpha) \lambda \tau \in H(M) .
$$

Then $\partial_{M}^{\prime}$ satisfies the conditions (5.6). If $\sigma$ is a cell of $K(F, \Phi)$, then $\alpha \in \Phi$, and since $\lambda$ is an operator homomorphism, $\partial_{F}{ }^{\prime} \sigma=H(\alpha) \lambda \tau=\lambda K(\alpha) \tau=\lambda \sigma$. Thus $\partial_{F}{ }^{\prime}$ is identical with $\lambda$ for the subgroup $C_{1}(K(F, \Phi))$ of $C_{1}(K(F))$, and condition (5.7) holds. If $\rho$ is any 2-cell of $K(M)$, with the canonical representation $K(\gamma) \eta$, for $\eta$ a typical 2-cell in $K(F, \Phi)$, then $\partial \rho=K(\gamma) \partial \eta$, so that by the naturality condition (5.6), one has

$$
\partial_{M}^{\prime} \partial \rho=\partial_{M}^{\prime} K(\gamma) \partial \eta=K(\gamma) \partial_{F}^{\prime} \partial \eta=K(\gamma) \lambda \partial \eta=0,
$$

by the assumption as to the kernel of $\lambda$. Therefore $\partial_{M}^{\prime} \partial=0$, as in (5.5), and $\partial_{M}^{\prime}$ provides an augmentation $\bar{K}$ of $K$. Since $\partial_{F}^{\prime}$ is identical with $\lambda$ on $C_{1}(K(F, \Phi))$ and thus has kernel $B_{1}(K(F, \Phi))$ and image $H(F, \Phi)$ $=C_{0}(\bar{K}(F, \Phi))$, it follows at once that the homology groups of $\bar{K}(F, \Phi)$ are zero in dimensions 0 and 1 . With condition (6.7) this shows that $\bar{K}$ is $\Phi$-acyclic.

In view of this theorem, it is natural to say that a $\Phi$-construction $K$ is $\Phi$-acyclic if it satisfies conditions (6.6) and (6.7). For each choice of an operator isomorphism realizing (6.6) there is a corresponding $\Phi$-acyclic augmentation $\bar{K}$ of $K$. 
TheOREM 6.5. Any two $\Phi$-acyclic constructions $K$ and $L$ for the same $F$ and $\Phi$ are chain equivalent.

Proof. Augment $K$ and $L$ to $\bar{K}$ and $\bar{L}$, as in Theorem 6.4. Then $\bar{K}$ and $\bar{L}$ are chain equivalent by Theorem 6.2 , and this induces a chain equivalence of the constructions $K$ and $L$. Note that the maps $f: K \rightarrow L$ and $g: L \rightarrow K$ will depend upon the choice of an operator isomorphism of $H_{1}(K(F, \Phi))$ to $H_{1}(L,(F, \Phi))$.

REMARK. The use of an augmentation has exactly the effect of imposing initial conditions upon constructions $K$, which have not otherwise been subjected to any initial conditions. It is possible to make other choices for initial conditions. In fact, suppose that we have any covariant functor [2] $T$ on $\mathscr{X}(F)$ to the category of abelian groups, and suppose further that in the group $T(F)$ we have a subgroup $T(F, \Phi)$ which is mapped into itself by the endomorphisms induced by the elements of $\Phi$. Then in the definition of an augmented construction we may replace $H(M)$ by $T(M)$ and $H(F, \Phi)$ by $T(F, \Phi)$. With this replacement, the theorems of this section all remain valid, except for the existence assertion of Theorem 6.2. The difficulty here is caused only by the fact that the proof of Lemma 6.3 utilized the special homomorphism $\mu_{F}: F \rightarrow H(F)$. The existence assertion may nevertheless be proved if we require that $T$ have the following property (which is essentially a portion of the conclusion of Lemma 6.3): For each $c$ in $T(F, \Phi)$, there is an integer $k$ such that the projection $\theta_{k}: F \rightarrow F$, with $\theta_{k}\left(g_{i}\right)=g_{i}, i=1, \cdots, k$, and $\theta\left(g_{j}\right)=1$ for $j>k$, satisfies $T\left(\theta_{k}\right) c=c$. The tensor product of $H(M)$ with itself appears to be one of the useful examples of such a functor $T$.

7. Constructions on the category $\mathcal{X}_{A}$. We consider the construction $A^{0}$ on the category $\mathcal{X}_{A}$ of associative systems as given in the first paragraph of the introduction. This construction is augmented by the homomorphisms $\partial_{M}^{\prime}: C_{0}\left(A^{0}(M)\right) \rightarrow H(M)$ defined by $\partial_{M}^{\prime}[x]=\mu_{M}(x)$ where $\mu_{M}: M \rightarrow H(M)$ is the natural map of $\S 3$.

The agumented construction $\bar{A}^{0}$ is a $\Phi$-construction for $\Phi=\Phi^{0}$ and $\Phi=\Phi^{*}$. We shall show that it is $\Phi$-acyclic for both choices of $\Phi$. We may also regard $\bar{A}^{0}$ as a construction on the category $\mathcal{X}_{G}$ of groups. Again it is freely, generically, and pseudo-generically acyclic.

Let $F_{A}$ be the free $A$-system with generators $g_{1}, g_{2}, \cdots, g_{k}, \cdots$. Then $H\left(F_{A}\right)=H\left(F_{A}, \Phi^{*}\right)$ is the free abelian group with generators $g_{k}^{\prime}=\mu_{F_{A}}\left(g_{k}\right)$, $k=1,2, \cdots$. Following Lyndon [4], we define an operator $E: C_{q}\left(\bar{A}^{0}\left(F_{A}\right)\right)$ $\rightarrow C_{q+1}\left(\bar{A}^{0}\left(F_{A}\right)\right)$ as follows:

$$
\begin{aligned}
E g^{\prime} & =[g] \quad(q=0), \\
E\left[1, x_{2}, \cdots, x_{q}\right] & =\left[1,1, x_{2}, \cdots, x_{q}\right], \\
E\left[g y, x_{2}, \cdots, x_{q}\right] & =-\left[g, y, x_{2}, \cdots, x_{q}\right]+E\left[y, x_{2}, \cdots, x_{q}\right],
\end{aligned}
$$


where $g$ is any one of the generators $g_{k}$. These formulas give $E$ by induction on the length of the first entry.

We verify that the relation

$$
\partial E c+E \partial c=c
$$

holds for every chain $c$. Thus if $\partial c=0$, then $\partial E c=c$. Observe further that if $c$ is generic, then $E c$ also is generic. This implies

$$
H_{q}\left(\bar{A}^{0}\left(F_{A}\right)\right)=H_{q}\left(\bar{A}^{0}\left(F_{A}, *\right)\right)=0,
$$

thus showing that $\bar{A}^{0}$ is freely and generically acyclic.

Now let $F_{G}$ be the free group with generators $g_{1}, g_{2}, \cdots, g_{k}, \cdots$. Then as before $H\left(F_{G}\right)=F_{G} /\left[F_{G}, F_{G}\right]$ is the free abelian group with generators $g_{i}^{\prime}=\mu_{F_{G}}\left(g_{i}\right)$. We supplement the definition of $E$ by setting

$$
E\left[g^{-1} y, x_{2}, \cdots, x_{q}\right]=\left[g, g^{-1} y, x_{2}, \cdots, x_{q}\right]+E\left[y, x_{2}, \cdots, x_{q}\right] .
$$

A computation then shows that

$$
E\left[y, x_{2}, \cdots, x_{q}\right]=E\left[g^{-1} g y, x_{2}, \cdots, x_{q}\right]=E\left[g g^{-1} y, x_{2}, \cdots, x_{q}\right],
$$

so that $E$ is defined without ambiguity. One may also show that the relation (7.1) still holds; hence it follows that $\bar{A}^{0}$, as a construction on the category of groups $\mathcal{X}_{G}$, is freely, generically, and pseudogenerically acyclic. The same operator $E$ also allows one to prove that the cohomology groups $H^{q}\left(F_{G}, C\right)$ are trivial for $q>1$ in case $F_{G}$ operates on the coefficient group $C$ on the right. In order to prove the same fact in case $F_{G}$ operates on $C$ on the left, one replaces [cf. 4] $E$ by a similar operator which acts on the last entry $x_{n}$ instead of the first one $x_{1}$.

8. Normalizations. In further illustrations of explicit constructions it will prove convenient to use constructions which are $\Phi$-acyclic only modulo suitable subconstructions. The objective of this section and the next is the proof that the use of such normalizations does not alter the content of the $\Phi$-acyclic homology theory. For this purpose, however, we must restrict the free systems $F$ considered to be either free groups or one of the free systems $F_{R}$, with $R$ a generic relation. Here a generic relation is a set of generic identities, where a generic identity is a pair $\left(w_{1}, w_{2}\right)$ of generic words of $F_{I}$ which involve exactly the same letters. In particular, the relations $I, A, C$, and $A C$ are generic. The force of this restriction is indicated by the following result.

LEMMA 8.1. If the free system $F$ with generators $g_{1}, g_{2}, \cdots$ is either one of the free groups $F_{G}$ and $F_{G A}$ or is $F_{R}$, for $R$ a generic relation, then $H(F)$ is a free abelian group with generators $\mu_{F}\left(g_{i}\right)$ while $H(F, \Phi)$ is identical with $H(F)$ for any admissible set $\Phi$ of endomorphisms.

Proof. For the free groups, the result is immediate. Let $R$ be a generic relation and $F_{I}$ the universal free system with generators $g_{1}^{\prime}, g_{2}^{\prime}, \cdots$, while 
$F_{0}$ is the free abelian group with generators $h_{1}, h_{2}, \cdots$, and $\omega: F_{I} \rightarrow F_{0}$, the homomorphism with $\omega\left(g_{i}^{\prime}\right)=h_{i}$ for all $i$. Let $S$ denote the set of all those identities $(u, v)$ in the closure $\bar{R}$ of the generic relation $R$ with the property that $\omega(u)=\omega(v)$. Then, by the definition of a generic relation, $R \subset S$, and $S$ is closed. Hence $S=\bar{R}$. Since $F_{R}$ is defined as $F_{I}$ modulo the equivalence relation $u \equiv v$ for $(u, v) \in \bar{R}$, it follows that $\omega$ induces a homomorphism $\omega^{\prime}: F_{R}$ $\rightarrow F_{0}$ with $\omega^{\prime}\left(g_{i}\right)=h_{i}$.

Now let $\alpha: F_{R} \rightarrow G$ be any homomorphism of $F_{R}$ into an abelian group $G$. Then there exists a homomorphism $\beta: F_{0} \rightarrow G$ with $\beta\left(h_{i}\right)=\alpha\left(g_{i}\right)$ for all $i$. The composite homomorphism $\beta \omega^{\prime}: F_{R} \rightarrow G$ then has $\beta \omega^{\prime}\left(g_{i}\right)=\alpha\left(g_{i}\right)$; since every element of $F_{R}$ is a product of the generators $g_{i}$, it follows that $\beta \omega^{\prime}=\alpha$. This factorization is unique, for if $\alpha=\gamma \omega^{\prime}$, then $\alpha\left(g_{i}\right)=\gamma \omega^{\prime}\left(g_{i}\right)=\gamma\left(h_{i}\right)$, so that $\beta=\gamma$. This unique factorization of every $\alpha: F_{R} \rightarrow G$ by $\omega^{\prime}=F_{R} \rightarrow F_{0}$ shows that $F_{0}$ is isomorphic to $H\left(F_{R}\right)$ and that $\omega^{\prime}$ corresponds to $\mu_{F R}$, so that $H\left(F_{R}\right)$ is the free abelian group described above. Since it is generated by the images $\omega^{\prime}\left(g_{i}\right)$ and since the $g_{i}$ are $\Phi$-elements of $F$ for any $\Phi$, it follows that $H\left(F_{R}, \Phi\right)$ $=H\left(F_{R}\right)$ for all $\Phi$.

Let $K$ be a $\Phi$-construction on the category $\mathcal{H}(F)$. A normalization $K_{N}$ of $K$ is a family of subcomplexes $K_{N}(M) \subset K(M)$ satisfying the following condition for every $M_{1}$ and $M_{2}$ : if $\sigma_{1}=\left[x_{1}, \cdots, x_{r}\right]_{t}$ is a cell of $K_{N}\left(M_{1}\right)$ in which the entries $x_{e_{1}} \cdots x_{e_{h}}$ (and no others) are 1 , then any cell $\sigma_{2}=\left[y_{1}, \cdots, y_{r}\right]_{t}$ of the same type in $K\left(M_{1}\right)$ and with $y_{e_{1}}=\cdots=y_{e_{h}}=1$ is a cell of $K_{N}\left(M_{2}\right)$. This condition states in effect that the presence of a cell in $K_{N}$ is determined entirely by the pattern of its unit entries. It follows from this condition that if $\beta: M_{1} \rightarrow M_{2}$, then $K(\beta)$ carries $K_{N}\left(M_{1}\right)$ into $K_{N}\left(M_{2}\right)$.

A normalization of an augmented construction $\bar{K}$ is a normalization of the associated construction $K$, subject to the additional condition that the augmenting homomorphisms $\partial_{M}^{\prime}: C_{1}(K(M)) \rightarrow H(M)$ each map $C_{1}\left(K_{N}(M)\right)$ into zero. For each $M$, the subcomplex $K_{N}(M)$ of $K(M)$ may then be regarded as a subcomplex $\bar{K}_{N}(M)$ of the augmented $\bar{K}(M)$, for the group of zero-dimensional chains of $K_{N}(M)$ is 0 .

We now list some examples of normalizations.

Zero normalization. A cell $\sigma$ of $K(M)$ is called a zero-cell if all the entries of $\sigma$ are 1 . In particular, every cell with no entries is a zero-cell. The zero-cells form a subcomplex $K_{0}(M)$ isomorphic with the complex $K\left(M_{0}\right)$ where $M_{0}$ is the system consisting of the elements 1 alone. The complexes $K_{0}(M)$ form a normalization $K_{0}$ of $K$, called the zero normalization. This is also valid for any augmentation.

Full normalization. Consider the cells which have no entries or in which at least one entry is 1 . If these cells form a subcomplex of each $K(M)$, then the resulting normalization of $K$ is denoted by $K_{1}$. In the construction $A_{0}$ the full normalization does yield a subcomplex. However, there are constructions for which this is not the case. 
Void normalization. For formal reasons we also introduce for any construction $K$ the void normalizations in which $K_{N}(M)$ is the empty subcomplex of $K(M)$. This normalization remains valid under any augmentation.

Let $\bar{K}$ be an augmented $\Phi$-construction on $\mathcal{H}(F)$ with a normalization $K_{N}$. We shall say that $K$ is $\Phi$-acyclic modulo $K_{N}$ provided that

$$
H_{q}\left(\bar{K}(F, \Phi), \bar{K}_{N}(F, \Phi)\right)=0, \quad q=0,1, \cdots,
$$

where $\bar{K}_{N}(F, \Phi)=\bar{K}(F, \Phi) \cap \bar{K}_{N}(F)$ is the cell complex spanned by the cells common to $\bar{K}(F, \Phi)$ and $\bar{K}_{N}(F)$.

THEOREM 8.2. If $\bar{K}$ is a $\Phi$-acyclic augmented construction on $\mathcal{H}(F)$, then $\bar{K}$ is also $\Phi$-acyclic modulo the zero normalization. Furthermore, the complexes $K_{0}(M)$ have trivial homology groups, while the homology groups of $K(M)$ are isomorphic, in the natural fashion, with those of $K(M)$ modulo $K_{0}(M)$.

Proof. We first show that the complexes $K_{0}(F, \Phi)$ and $K_{0}(M)$ have trivial homology groups. Let $z \in Z_{q}\left(K_{0}(F, \Phi)\right)$. Then $z \in Z_{q}(\bar{K}(F, \Phi))$. Hence $z=\partial c$, where $c \in C_{q+1}(K(F, \Phi))$. Let $\alpha: F \rightarrow F$ be that endomorphism which maps all generators into 1 . Then $z=K(\alpha) z=K(\alpha) \partial c=\partial K(\alpha) c$ and $K(\alpha) c$ $\in C_{q+1}\left(K_{0}(F, \Phi)\right)$. This proves that $K_{0}(F, \Phi)$ has trivial homology groups. Since $K_{0}(F, \Phi)$ and $K_{0}(M)$ are isomorphic, the same follows for $K_{0}(M)$. The natural homomorphism $H_{q}(K(M)) \rightarrow H_{q}\left(K(M), K_{0}(M)\right)$ is then an isomorphism onto by the exactness of the relative homology sequence. Condition (8.1) also follows by exactness.

Let $K$ and $L$ be two $\Phi$-constructions, with normalizations $K_{N}$ and $L_{N}$, defined on the same category $\mathcal{H}(F)$. A map $f: K / K_{N} \rightarrow L / L_{N}$ is a set of homomorphisms

such that:

$$
f_{M}: C_{q}(K(M)) \rightarrow C_{q}(L(M)), \quad M \in \mathcal{M}(F),
$$

(8.2) $f_{M}\left[C_{q}\left(K_{N}(M)\right)\right] \subset C_{q}\left(L_{N}(M)\right), q=1,2, \cdots$

(8.3) $\partial f_{M} \equiv f_{M} \partial \bmod C_{q-1}\left(L_{N}(M)\right), q=2, \cdots \cdot$

(8.4) If $\alpha: M_{1} \rightarrow M_{2}$, then $f_{M_{2}} K(\alpha)=L(\alpha) f_{M_{1}}$.

(8.5) The map $f_{F}$ carries $K(F, \Phi)$ into $L(F, \Phi)$.

A homotopy $D: f^{0} \simeq f^{\prime}$ of two maps $f^{0}, f^{1}: K / K_{N} \rightarrow L / L_{N}$ is a family of homomorphisms

$$
D_{M}: C_{q}(K(M)) \rightarrow C_{q+1}(L(M)), \quad M \in \mathcal{H}(F),
$$

such that:

(8.7) $D_{M}\left[C_{q}\left(K_{N}(M)\right)\right] \subset C_{q+1}\left(L_{N}(M)\right)$.

(8.8) $\partial D_{M}+D_{M} \partial \equiv f^{1}-f^{0} \bmod C_{q}\left(L_{N}(M)\right)$.

(8.9) If $\alpha: M_{1} \rightarrow M_{2}$, then $D_{M_{2}} K(\alpha)=L(\alpha) D_{M_{1}}$.

(8.10) The homotopy $D_{F}$ carries $K(F, \Phi)$ into $L(F, \Phi)$.

For maps $f: \bar{K} / \bar{K}_{M} \rightarrow \bar{L} / \bar{L}_{N}$ of augmented constructions with normalizations, we further require that 
(8.11) $\partial_{M}^{\prime} c=\partial_{M}^{\prime} f_{M} c$ for each $c \in C_{1}(K(M))$.

Under this condition, $f_{M}$ may be extended to a map $\bar{f}_{M}$ of $\bar{K}(M) / \bar{K}_{N}(M)$ into $\bar{L}(M) / \bar{L}_{M}(M)$, and any homotopy may be similarly augmented.

Given any finite set of integers $A$ we shall denoted by $\eta_{A}$ the endomorphism $\eta_{A}: F \rightarrow F$ such that $\eta_{A} g_{i}=1$, for $i \in A$, and $\eta_{A} g_{i}=g_{i}$ otherwise. If $A$ reduces to a single integer $n$, we write $\eta_{A}=\eta_{n}$. Clearly $\eta_{A} \eta_{A}=\eta_{A}$, and $\eta_{A} \eta_{B}$ $=\eta_{A} \cup{ }_{B}=\eta_{B} \eta_{A}$.

LEMMA 8.3. Let $\bar{L}$ be an augmented $\Phi$-construction with a normalization $L_{N}$. Then $\bar{L}$ is $\Phi$-acyclic modulo $L_{N}$ if and only if there is a sequence of homomorphisms

$$
R_{q}: C_{q}(\bar{L}(F, \Phi)) \rightarrow C_{q+1}(\bar{L}(F, \Phi)), \quad q=0,1, \cdots,
$$

such that:

$$
\begin{aligned}
R_{q}\left[C_{q}\left(L_{N}(F, \Phi)\right)\right] & \subset C_{q+1}\left(L_{N}(F, \Phi)\right), \\
\partial R_{q} c+R_{q-1} \partial c & \equiv c\left(\bmod C_{q}\left(L_{N}(F, \Phi)\right)\right), \\
R_{q} L\left(\eta_{A}\right) & =L\left(\eta_{A}\right) R_{q},
\end{aligned}
$$

all $A$.

Proof. Sufficiency is a direct consequence of (8.12) and (8.13). Conversely, we begin by constructing homomorphisms $\left\{R_{q}^{0}\right\}$ satisfying conditions (8.12) and (8.13) but not necessarily condition (8.14).

To define $R_{0}^{0}$ we observe that, by Lemma $8.1, H(F, \Phi)=H(F)$ is the free group generated by $g_{i}^{\prime}=\mu_{F}\left(g_{i}\right)$, where the $g_{i}$ are the generators of $F$. Since $H_{0}\left(\bar{L}(F, \Phi), \bar{L}_{N}(F, \Phi)\right)=0$, the mapping $\partial_{F}^{\prime}$ carries $C_{1}(\bar{L}(F, \Phi))$ onto $H(F, \Phi)$ $=C_{0}(\bar{L}(F, \Phi))$, hence there exists a homomorphism $R_{0}^{0}$ of the free group $H(F, \Phi)$ into $C_{1}\left(L\left(F_{1}, \Phi\right)\right)$ such that $\partial_{F}^{\prime} R_{0}^{0}$ is the identity, as required in (8.13).

Suppose now that $R_{a}^{0}$ is already defined for all $q<m$, where $m>0$, and let $\sigma$ be a $\Phi$-cell of dimension $m$. Then if $\sigma \in L_{N}(F, \Phi)$, define $R_{m}^{0} \sigma=0$, and (8.12) and (8.13) hold. For $\sigma$ not in $L_{N}$ consider

$$
\partial\left(\sigma-R_{m-1}^{0} \sigma\right)=\partial \sigma-\partial R_{m-1}^{0} \sigma \equiv \partial \sigma-\left(\partial \sigma-R_{m-2}^{0} \partial \partial \sigma\right) \equiv 0 .
$$

Since $H_{m}\left(L(F, \Phi), L_{N}(F, \Phi)\right)=0$, there is an $(m+1)$ chain $R_{m}^{0} \sigma$ in $L(F, \Phi)$ with $\partial R_{m}^{0} \sigma \equiv \sigma-R_{m-1}^{0} \partial \sigma$. Then (8.12) and (8.13) hold. This concludes the definition of $\left\{R_{q}^{0}\right\}$ satisfying (8.12) and (8.13).

We now define the homomorphisms $R_{q}^{n}$ by induction with respect to $n$ as follows:

$$
R_{q}^{n}=R_{q}^{n-1}-L\left(\eta_{n}\right) R_{q}^{n-1}-R_{q}^{n-1} L\left(\eta_{n}\right)+2 L\left(\eta_{n}\right) R_{q}^{n-1} L\left(\eta_{n}\right) .
$$

It is then easy to see that $R_{q}^{n}$ still satisfy (8.12) and (8.13) and that

$$
R_{q}^{n} L\left(\eta_{i}\right)=L\left(\eta_{i}\right) R_{q}^{n}, \quad i \leqq n .
$$


For each chain $c$ there is by Lemma 6.3 an integer $k$ with the corresponding projection $\theta_{k}, \theta_{k}\left(g_{i}\right)=g_{i}, i=1, \cdots, k, \theta\left(g_{j}\right)=1, j>k$, such that both $\bar{L}\left(\theta_{k}\right) c=c$ and $\bar{L}\left(\theta_{k}\right) R_{q}^{0} c=R_{q}^{0} c$. By induction on $n$, it follows that $\bar{L}\left(\theta_{k}\right) R_{q}^{n} c=R_{q}^{n} c$. For $n>k, \theta_{k} \eta_{n}=\theta_{k}$ and hence $R_{q}^{n+1} c=R_{q}^{n} c$. Thus setting $R_{q}=\lim (n \rightarrow \infty) R_{q}^{n}$ yields the desired homomorphisms.

\section{Main theorem for normalization.}

TheOREM 9.1. Let $\bar{K}$ and $\bar{L}$ be two augmented $\Phi$-constructions on the same category $\mathcal{X}(F)$ with normalizations $K_{N}$ and $L_{N}$, respectively. If $\bar{L}$ is $\Phi$-acyclic modulo $L_{N}$, there exist maps $f: \bar{K} / K_{N} \rightarrow \bar{L} / L_{N}$, and any two such maps are homotopic.

The mapping $\bar{f}_{M}$ in dimension 0 is chosen as the identity, and is then defined inductively as follows. Let $\sigma$ be any $q$-cell of $K(M)$, for $q>0$, and $\sigma=K(\alpha) \tau$ its canonical representation. Then set

$$
f_{M} \sigma=L(\alpha) R_{q-1} f_{F} \partial \tau,
$$

where $R_{q-1}$ is the homotopy for $L$ given in Lemma 8.3. To verify (8.2) assume that $\sigma=\left[x_{1}, \cdots, x_{n}\right]_{t}$ is in $K_{N}(M)$. Let $A$ be the set of indices at which $\sigma$ has entries 1 . Then $\tau=\left[g_{1}, \cdots, g_{n}\right]_{t}$ and $\alpha \eta_{A}=\alpha$. Thus $\sigma=K(\alpha) \tau=K(\alpha) K\left(\eta_{A}\right) \tau$, and $K\left(\eta_{A}\right) \tau$ is in $K_{N}(F, \Phi)$. Then

$$
\begin{aligned}
f_{M} \sigma & =L(\alpha) R_{q-1} f_{F} \partial \tau=L(\alpha) L\left(\eta_{A}\right) R_{q-1} f_{F} \partial \tau \\
& =L(\alpha) R_{q-1} L\left(\eta_{A}\right) f_{F} \partial \tau=L(\alpha) R_{q-1} f_{F} \partial K\left(\eta_{A}\right) \tau .
\end{aligned}
$$

Since $K\left(\eta_{A}\right) \tau$ is in $K_{N}(F, \Phi)$ it follows that $R_{q-1} f_{F} \partial K\left(\eta_{A}\right) \tau$ is in $L_{N}(F, \Phi)$, and $f_{M} \sigma$ is in $L_{N}(M)$. The proof of (8.3), (8.4), (8.5), and (8.11) is purely formal and is left to the reader.

The definition of the homotopy $D: f^{0} \simeq f^{1}$ required for Theorem 9.1 is given by the following inductive formulas,

$$
\begin{aligned}
D c & =0, & c \in C_{0}(\bar{K}(M)), \\
D_{M} \sigma & =L(\alpha) R_{q}\left(f_{F}^{1} \tau-f_{F}^{0} \tau-D_{F} \partial \tau\right), &
\end{aligned}
$$

where $\sigma=K(\alpha) \tau$ is the canonical representation of the 4 -cell $\sigma$ of $K(M)$ $(q \geqq 1)$. The proof of $(8.7)-(8.10)$ is left to the reader.

Theorem 9.2. Any two augmented $\Phi$-constructions $\bar{K}$ and $\bar{L}$ on the same category $\operatorname{SC}(F)$ which are $\Phi$-acyclic modulo normalization $K_{N}$ and $L_{N}$, respectively, are chain equivalent, modulo $K_{N}$ and $L_{N}$.

The proof is immediate. In particular, since $L_{N}$ could be the void normalization, it follows that if the agumented $\Phi$-construction $\bar{K}$ is $\Phi$-acyclic, modulo a normalization $K_{N}$, then $\bar{K} / K_{N}$ does yield the $\Phi$-acyclic homology theory of $\mathscr{H}(F)$. 
THEOREM 9.3. $A \Phi$-construction $K$ with a normalization $K_{N}$ can be augmented to a $\Phi$-construction $\bar{K}$ generically acyclic modulo $K_{N}$ if, and only if,

(9.1) $H_{1}\left(K(F, \Phi), K_{N}(F, \Phi)\right)$ is operator isomorphic to $H(F, \Phi)$, for all operators $\alpha \in \Phi$,

(9.2) $H_{q}\left(K(F, \Phi), K_{N}(F, \Phi)\right)=0, q=2, \cdots$.

The proof is analogous to that of Theorem 6.4. The operator homomorphism $\lambda: C_{1}(K(F, \Phi)) \rightarrow H(F, \Phi)$ given by (9.1) now has kernel $B_{1}(K(F, \Phi))$ $\cup C_{1}\left(K_{N}(F, \Phi)\right)$. The only new observation needed is the proof that $\partial_{M}^{\prime}$ maps $C_{1}\left(K_{N}(M)\right)$ into zero, which is a consequence of the fact that $\lambda$ maps $C_{1}\left(K_{N}(F, \Phi)\right)$ to zero and the definition of a normalization by means of "positions" of 1 's.

The construction $A^{0}$ for associative systems as given in $\$ 7$ admits both the zero normalization $A_{0}^{0}$ and the full normalization $A_{1}^{0}$. The operator $E$ constructed in $\$ 7$ carries $A_{1}^{0}$ into $A_{1}^{0}$, thus showing that $\bar{A}^{0}$ is both freely and generically acyclic modulo $A_{1}^{0}$. Thus the homology theory of any system $M \in \mathcal{X}_{A}$ may be obtained by using any one of the complexes $A^{0}(M)$, $A^{0}(M) / A_{0}^{0}(M)$, or $A^{0}(M) / A_{1}^{0}(M)$. The same remark applies to the category $\mathcal{X}_{G}$ of groups, for free, generic, and pseudo-generic acyclicity.

Remark. To follow up the remark at the end of $\$ 6$ as to alternative choices of the augmentation, it should be noted that the results of these two sections on normalizations are valid for agumentation by any functor $T$ as described in the remark, provided that $T(F, \Phi)$ is a free abelian group.

10. Constructions on the categories $\mathscr{X}_{I}$ and $\mathscr{X}_{C}$. We consider the following construction $K$ on the category $\mathscr{X}_{I}=\mathscr{X}\left(F_{I}\right)$ of all multiplicative systems:

1-cells: $[x]$.

2-cells: $[x, y]$ with $\partial[x, y]=[y]-[x y]+[x]$.

We augment this construction by setting $\partial_{M}^{\prime}[x]=\mu_{M} x$, where $\mu_{M}: M \rightarrow H(M)$ is the natural map of $\S 3$. The construction $K$ thus obtained clearly is an augmented generic construction. In $\bar{K}$ we consider the normalization subcomplex $K_{1}$ given by the cells $[1],[1, y]$, and $[x, 1] ; K_{1}$ is thus the full normalization. We shall show that $\bar{K} \bmod K_{1}$ is both freely and generically acyclic.

The universal free system $F_{I}$ with generators $g_{1}, g_{2}, \cdots, g_{k}, \cdots$, has $H\left(F_{I}\right)=H\left(F_{I}, *\right)_{\text {, which }}$ is the free abelian group with free generators $g_{k}^{\prime}$ $=\mu_{F_{I}}\left(g_{k}\right)$. We define an operator $E: C_{q}\left(\bar{K}\left(F_{I}\right)\right) \rightarrow C_{q+1}\left(\bar{K}\left(F_{I}\right)\right)$ as follows:

$$
\begin{aligned}
E g^{\prime} & =[g], \quad E[1]=0, \quad E[g]=0, \quad g \text { a generator, } \\
E[x y] & =E[x]+E[y]-[x, y], \quad \text { for } x \neq 1, y \neq 1, \\
E[x, y] & =0,
\end{aligned}
$$

and observe that $E$ maps $K_{1}\left(F_{I}\right)$ into $K_{1}\left(F_{I}\right)$. The relation

$$
\partial E c+E \partial c \equiv c\left(\bmod K_{1}\left(F_{I}\right)\right)
$$


may readily be verified for each chain $c$. In particular, if $\partial c \equiv 0$, then $c \equiv \partial E c$. This proves that $\bar{K} \bmod K_{1}$ is freely acyclic. Observe further that if $c$ is generic, then $E c$ also is generic, thus $\bar{K} \bmod K_{1}$ also is generically acyclic.

The construction $K$ on $\mathscr{X}_{I}$ is not acyclic (freely or generically) without the normalization $K_{1}$. Indeed $Z=[x, 1]-[1,1]$ and $Z^{\prime}=[1, x]-[1,1]$ are nonbounding 2-cycles. This necessitates 3-cells $[x]_{3}$ and $[x]_{3}^{\prime}$ with $\partial[x]_{3}=Z$, $\partial[x]_{3}^{\prime}=Z^{\prime}$. Then $[1]_{3}$ and $[1]_{3}^{\prime}$ are nonbounding cycles causing the introduction of 4 -cells []$_{4}$ and []$_{4}^{\prime}$ with zero entries and with $[1]_{3}$ and $[1]_{3}^{\prime}$, respectively, as boundaries. The resulting modified construction $K^{\prime}$ is generically and freely acyclic without any normalization. This shows that the use of normalizations cuts down the number of types of cells necessary in the various constructions.

The augmented construction $\bar{K}$ described above may also be applied to any category $\mathcal{X}(F)$ where $F$ is $F_{G}, F_{G A}$, or one of the free systems $F_{R}$ with $R$ a generic relation. By Lemma 8.1, $H(F)$ is then a free abelian group, and the definition for $E$ will still apply (writing $E\left[g^{-1}\right]=0$ in the free group cases). The relation $\partial E c+E \partial c=c$ remains valid for chains $c$ of dimensions 0 or 1 . Thus $\bar{K}$, regarded as a construction on $\mathcal{H}(F)$, and with or without the full normalization, is generically and freely acyclic in dimensions 0 and 1 . The argument of the existence theorem will then enlarge $\bar{K}$ to a construction freely or generically acyclic on $\mathcal{X}(F)$, by the adjunction of cells of dimension at least 3 . Thus $K$ may serve as the starting point for many constructions.

The homology group $H_{1}(M)$ computed using the construction $K$ is the group $H(M)$ of $\S 3$. The cohomology group $H^{1}(M ; G)$ for any coefficient group $G$ is the group of all homomorphisms $f: M \rightarrow G$. This is valid for any $M \in \mathcal{X}(F)$.

The cohomology group $H^{2}(M ; G)$ for $M \in \mathscr{X}_{I}$ calculated using the construction $K$ is the group of all functions $f: M \times M \rightarrow G$ satisfying $f(x, 1)=0$, $f(1, y)=0$, reduced modulo functions of the form

$$
f(x, y)=h(y)-h(x y)+h(x),
$$

for some $h$ with $h(1)=0$. For any $M \in \mathcal{X}_{R}$ the cohomology group $H^{2}(M ; G)$ calculated using a generically or freely acyclic construction on $\mathcal{X}_{R}$ will be isomorphic to a subgroup of the group $H^{2}(M ; G)$ described above.

Now we pass to the study of the category $\mathcal{X}_{C}$ of commutative systems. We consider the following generic construction $K$ :

1-cells: $[x]$.

2-cells: $[x, y]$ with $\partial[x, y]=[y]-[x y]+[x]$.

$n$-cells $(n>2):[x, y]_{n}$ with $\partial[x, y]_{n}=[x, y]_{n-1}+(-1)^{n}[y, x]_{n-1}$.

In the last formula $[x, y]_{2}$ is to be interpreted as $[x, y]$. The proof that $\partial \partial[x, y]_{3}=0$ requires the use of the commutative law. For other cells the proof that $\partial \partial=0$ is purely formal. As in the earlier construction we augment $K$ by setting $\partial_{M}^{\prime}[x]=\mu_{M}(x)$. In the augmented construction $\bar{K}$ we consider the full normalization $K_{1}$. 
We also consider the (nongeneric) construction $L$ obtained from $K$ by adjoining:

$n$-cells $(n>3):[x]_{n}$ with $\partial[x]_{n}=[x, x]_{n-1}-\left(1+(-1)^{n-1}\right)[x]_{n-1}$.

The construction $L$ is augmented just as $K$ was, and $L_{1}$ denotes the full normalization of $L$.

Let $F_{C}$ be the free commutative system with generators $g_{1}, g_{2}, \cdots$, $g_{k}, \cdots$, as defined in $\S 2$. We order the elements of $F_{C}$ so that $1<x$ for all $x \neq 1$. It follows then from the construction of $F_{C}$ that every element $x$ of $F_{C}$ which is not 1 or a generator can be written uniquely as a product $x=y z$ with $y \leqq z, y \neq 1, z \neq 1$. If $x$ is generic, then $y$ and $z$ must be generic and disjoint and thus $y<z$.

By Lemma 8.1, $H\left(F_{C}\right)=H\left(F_{C}, *\right)$ is the free abelian group with free generators $g_{k}^{\prime}=\mu_{F_{C}}\left(g_{k}\right)$.

Now we define the operator $E: C_{q}\left(\bar{L}\left(F_{C}\right)\right) \rightarrow C_{q+1}\left(\bar{L}\left(F_{C}\right)\right)$, as follows: $E c=0$, for $c \in L_{1}\left(F_{c}\right)$, and otherwise

$$
\begin{aligned}
& E g^{\prime}=[g], \quad E[g]=0, \\
& E[x y]=E[x]+E[y]-[x, y] \text {, } \\
& E[x, y]=\left\{\begin{array}{l}
{[x, y]_{3},} \\
0,
\end{array}\right. \\
& E[x, y]_{n}=\left\{\begin{array}{l}
{[x, y]_{n+1},} \\
0,
\end{array}\right. \\
& E[x, x]_{n}=[x]_{n+1}, \\
& E[x]_{n}=0 .
\end{aligned}
$$

A direct computation shows that

$$
\partial E c+E \partial c \equiv C\left(\bmod L_{1}\left(F_{C}\right)\right),
$$

which proves that $\bar{L} \bmod L_{1}$ is freely acyclic. We notice further that the same operator $E$ maps the generic subcomplex $\bar{K}\left(F_{C}, *\right)$ of the construction $\bar{K}$ into itself and therefore proves that the construction $\bar{K} \bmod K_{1}$ is generically acyclic.

These constructions yield easily a computation of the cohomology groups $H^{q}(M, G)$ over any coefficient group $G$, both in the generic and in the free theory. We tabulate the results.

In the generic theory:

$H^{1}(M, G)$ is the group of all homomorphisms $f: M \rightarrow G$.

$H^{2}(M, G)$ is the group of all functions $f: M \times M \rightarrow G$ satisfying

$$
f(x, 1)=0, \quad f(1, y)=0, \quad f(x, y)=f(y, x)
$$

reduced modulo functions of the form 


$$
f(x, y)=h(y)-h(x y)+h(x),
$$

where $h$ is a function on $M$ to $G$ with $h(1)=0$.

$H^{n}(M ; G)(n>2, n$ odd $)$ is isomorphic with the group of all functions $t: M \rightarrow G$ such that $t(1)=0$ and $2 t(x)=0$ for all $x \in M$. The correspondence is obtained by assigning, to each cocycle $f \in Z^{n}\left(K(M), K_{1}(M)\right.$; $\left.G\right)$, its trace $t(x)=f\left([x, x]_{n}\right)$.

$H^{n}(M ; G)(n>2, n$ even) is isomorphic with the group of all functions $t: M \rightarrow G / 2 G$ such that $t(1)=0$. The correspondence is obtained by assigning to each cocycle $f \in Z^{n}\left(K(M), K_{1}(M) ; G\right)$ its trace $t(x)=f\left([x, x]_{n}\right) \bmod 2 G$.

In the free theory the groups $H^{1}(M ; G)$ and $H^{2}(M ; G)$ are the same as in the generic theory, while the groups $H^{n}(M ; G)$ for $n>2$ are trivial.

\section{Additivity theorems for associative and commutative systems.}

THEOREM 11.1. The generically acyclic (and the freely acyclic) homology theory for the category $\mathcal{X}_{A C}$ (or $\mathcal{X}_{G A}$ ) give integral homology groups which satisfy the direct sum theorem:

$$
H_{q}\left(M+M^{\prime}\right)=H_{q}(M)+H_{q}\left(M^{\prime}\right),
$$

with the analogous results for cohomology groups.

For convenience, we write the composition in systems $M \in \mathcal{X}_{A C}$ as addition, and the unit element as 0 . The direct sum $M+M^{\prime}$, defined in the usual fashion as the set of pairs $\left(x, x^{\prime}\right)$ with $x \in M, x^{\prime} \in M^{\prime}$ and termwise addition, has projections

$$
\rho: M+M^{\prime} \rightarrow M, \quad \rho^{\prime}: M+M^{\prime} \rightarrow M^{\prime},
$$

and injections

$$
\lambda: M \rightarrow M+M^{\prime}, \quad \lambda^{\prime}: M^{\prime} \rightarrow M+M^{\prime},
$$

defined by $\rho\left(x, x^{\prime}\right)=x, \rho\left(x, x^{\prime}\right)=x^{\prime}, \lambda x=(x, 0), \lambda^{\prime} x^{\prime}=\left(0, x^{\prime}\right)$, which yield the direct sum diagram

$$
M \rightleftarrows M+M^{\prime} \leftrightarrows M^{\prime} .
$$

If $\rho_{*}$ and $\rho_{*}^{\prime}$ are the homomorphisms induced by the projections (11.2) on the homology groups, then the isomorphism (11.1) to be established is to be given by the correspondence

$$
h \rightarrow\left(\rho_{*} h, \rho_{*}^{\prime} h\right), \quad h \in H_{q}\left(M+M^{\prime}\right) .
$$

The presence of both associative and commutative laws allows the construction of the sum $\alpha+\alpha^{\prime}$ of two homomorphisms $\alpha, \alpha^{\prime}: M \rightarrow N$ as the homomorphism with $\left(\alpha+\alpha^{\prime}\right)(x)=\alpha(x)+\alpha^{\prime}(x)$. The direct sum diagram (11.4) has the properties 


$$
\begin{aligned}
& \lambda \rho+\lambda^{\prime} \rho^{\prime}=I: M+M^{\prime} \rightarrow M+M^{\prime}, \\
& \rho \lambda=I, \quad \rho^{\prime} \lambda^{\prime}=I, \quad \rho \lambda^{\prime}=0, \quad \rho^{\prime} \lambda=0,
\end{aligned}
$$

where $I$ and 0 denote the appropriate identity and zero homomorphisms; these properties characterize the diagram (11.6) up to isomorphism.

We construct the corresponding diagram

$$
H_{q}(M) \rightleftarrows H_{q}\left(M+M^{\prime}\right) \leftrightarrows H_{q}\left(M^{\prime}\right)
$$

with the induced homomorphisms $\lambda_{*}, \rho_{*}$, and so forth, on the homology groups; the result (11.1), under the explicit isomorphism (11.5), will follow if we can show the analogues of (11.6) and (11.7) for this diagram. The proof will depend on the method used in the uniqueness and normalization theorems above.

By Theorem 6.2 we may choose an augmented construction $\bar{K}$ on the category $\mathcal{X}_{A C}$ which is generically (freely) acyclic; the homology group $H_{q}(M)$ is then represented as $H_{q}(K(M))$ and the map $\alpha_{*}$ induced by any $\alpha$ as the map induced by $K(\alpha)$. In particular, an identity map $I$ induces an identity map $I_{*}$ on the homology groups. Since, by Theorem 8.2 , we may employ the zero normalization $K_{0}$, it follows that any zero homomorphism of $M_{1}$ in to $M_{2}$ induces the zero homomorphism of $H_{q}\left(M_{1}\right)$ into $H_{q}\left(M_{2}\right)$. These observations yield at once the analogue of (11.7)

$$
\rho_{*} \lambda_{*}=I, \quad \rho_{*}^{\prime} \lambda_{*}^{\prime}=I, \quad \rho_{*} \lambda_{*}^{\prime}=0, \quad \rho_{*}^{\prime} \lambda_{*}=0 .
$$

The analogue of (11.6) will also follow if we can establish a homotopy

$$
E: K(\lambda \rho)+K\left(\lambda^{\prime} \rho^{\prime}\right) \simeq I: K\left(M+M^{\prime}\right) \rightarrow K\left(M+M^{\prime}\right) .
$$

To this end, consider the direct sum $F+F^{\prime}$ of two free associative and commutative systems $F$ and $F^{\prime}$ with generators $g_{i}, g_{i}^{\prime}$, respectively, and write $\lambda_{0}, \rho_{0}, \lambda_{0}^{\prime}, \rho_{0}^{\prime}$ for the mappings involved in this direct sum diagram. This direct sum may also be considered as a single free system with $g_{1}, g_{1}^{\prime}, g_{2}, g_{2}^{\prime}, \ldots$ as its list of generators, hence the augmented complex $\bar{K}\left(F+F^{\prime}, \Phi\right)$ is acyclic. If $M+M^{\prime}$ and $M_{1}+M_{1}^{\prime}$ are any two direct sums, we consider the "special" homomorphisms $\beta: M+M^{\prime} \rightarrow M_{1}+M_{1}^{\prime}$ induced by a pair of homomorphisms $M \rightarrow M_{1}, M^{\prime} \rightarrow M_{1}^{\prime}$; for any such $\beta$ we have $\beta \lambda \rho=\lambda_{1} \rho_{1} \beta, \beta \lambda^{\prime} \rho^{\prime}$ $=\lambda_{1}^{\prime} \rho_{1}^{\prime} \beta$. As typical cells we now take the cells $\tau=\left[g_{1}+h_{1}, \cdots, g_{r}+h_{r}\right]_{t}$ of $K\left(F+F^{\prime}, \Phi\right)$; any cell $\sigma=\left[x_{1}+x_{1}^{\prime}, \cdots, x_{r}+x_{r}^{\prime}\right]_{t}$ of $K\left(M+M^{\prime}\right)$ then has a canonical representation $\sigma=K(\alpha) \tau$, with $\tau$ typical and $\alpha$ the special homomorphism $\alpha: F+F^{\prime} \rightarrow M+M^{\prime}$ with $\alpha g_{i}=x_{i}, \alpha g_{i}^{\prime}=x_{i}^{\prime}$ for $i=1, \cdots, r$ and $\alpha g_{j}=\alpha h_{j}=0$, for $j>r$.

The homotopy $E=E_{M, M}$, is now constructed by induction on dimension so as to satisfy the additional conditions

$$
K(\beta) E_{M, M^{\prime}}=E_{M_{1}, M_{1}^{\prime} K(\beta)}
$$


for any special $\beta: M+M^{\prime} \rightarrow M_{1}+M_{1}^{\prime}$, and

$$
E_{F, F^{\prime}} C_{q}\left(K\left(F+F^{\prime}, \Phi\right)\right) \subset C_{q+1}\left(K\left(F+F^{\prime}, \Phi\right)\right) .
$$

As in previous proofs, $E$ is first defined for typical cells, and then "carried around" by the canonical representation.

Before starting the induction, we observe that the group $H\left(F+F^{\prime}\right)$ used in augmenting $K$ is the free abelian group with free generators $\mu g_{i}, \mu g_{i}^{\prime}$, $i=1,2, \cdots$, and that the endomorphism $H\left(\lambda_{0} \rho_{0}\right)+H\left(\lambda_{0}^{\prime} \rho_{0}^{\prime}\right)$ of $H\left(F+F^{\prime}\right)$ is the identity.

For a typical cell $\tau$ of dimension 1 the chain

$$
c_{1}=\tau-K\left(\lambda_{0} \rho_{0}\right) \tau-K\left(\lambda_{0}^{\prime} \rho_{0}^{\prime}\right) \tau
$$

has boundary

$$
\partial^{\prime} c_{1}=\partial^{\prime} \tau-H\left(\lambda_{0} \rho_{0}\right) \partial^{\prime} \tau-H\left(\lambda_{0}^{\prime} \rho_{0}^{\prime}\right) \partial^{\prime} \tau=0 ;
$$

hence $c_{1}$ is a 1-cycle in $\bar{K}\left(F+F^{\prime}, \Phi\right)$, and therefore is the boundary of a 2chain $c_{2}$ of $K\left(F+F^{\prime}, \Phi\right)$. We set $E c_{1}=c_{2}$, and carry this definition around. In order to prove the essential property (11.11) for $q=1$, we must observe that if $\sigma=\left[a_{1}+b_{1}, \cdots, a_{r}+b_{r}\right]_{t}$ is a cell of $K\left(F+F^{\prime}, \Phi\right)$, then the mapping $\alpha$ in its canonical representation $\sigma=K(\alpha) \tau$ lies in $\Phi$. If $\Phi=\Phi^{0}$ (free acyclicity), this is immediate. If $\Phi=\Phi^{*}$ (generic acyclicity), then the elements $a_{1}, b_{1}, \cdots$, $a_{r}, b_{r}$ are indeed disjoint generic elements of $F+F^{\prime}$, with the generators $g_{1}, g_{1}^{\prime}, \cdots$, and again $\alpha \in \Phi^{*}$. (These arguments appear to break down if $\Phi$ is an arbitrary admissible set.)

The inductive construction of $E$ is continued exactly as in the previous cases. To obtain the analogous result for cohomology, one need change only the notation in (11.5), (11.8), and (11.7').

A more general related result is the following:

THEOREM 11.2. In the generically (or freely) acyclic homology theory for $\mathcal{X}_{A C}$ or $\mathcal{H}_{G A}$ the homomorphisms induced on homology groups by the sum of two homomorphisms $\alpha, \alpha^{\prime}: M \rightarrow N$ satisfy

$$
\left(\alpha+\alpha^{\prime}\right)_{*}=\alpha_{*}+\alpha_{*}^{\prime}: H_{q}(M) \rightarrow H_{\vartheta}(N) .
$$

This result includes the fact that $0_{*}=0$, for any zero homomorphism, and Theorem 11.1 can be proved formally from Theorem 11.2, using the direct sum diagrams.

Proof. We require a homotopy

$$
D: K(\alpha)+K\left(\alpha^{\prime}\right) \simeq K\left(\alpha+\alpha^{\prime}\right): K(M) \rightarrow K(N) .
$$

We use the diagram

$$
M \stackrel{\mu}{\rightarrow} M+M \stackrel{\alpha \times \alpha^{\prime}}{\rightarrow} N+N \stackrel{\nu}{\rightarrow} N,
$$


where $\mu(x)=(x, x),\left(\alpha \times \alpha^{\prime}\right)(x, y)=\left(\alpha x, \alpha^{\prime} y\right)$ and $\nu\left(z_{1}, z_{2}\right)=z_{1}+z_{2}$. Then

$$
\alpha+\alpha^{\prime}=\nu\left(\alpha \times \alpha^{\prime}\right) \mu,
$$

and $D$ may be constructed from the $E$ of (11.9) as

$$
D=K(\nu) K\left(\alpha \times \alpha^{\prime}\right) E K(\mu) .
$$

The requisite properties follow easily.

12. The cubical construction. An explicit generically acyclic construction $Q$ for the category $\mathscr{X}_{A C}$ will now be defined. For convenience, the composition for systems $M$ in $\mathscr{X}_{A C}$ will again be written as addition. In each dimension, the construction $Q(M)$ will have just one type of cell. In dimension 1, the cells are the cells $[x]$, for $x \in M$, and in dimension 2 the cells are all pairs $[x, y]$ of elements in $M$, with boundary

$$
\partial[x, y]=[x]+[y]-[x+y] .
$$

In dimension 3 , the cells are square arrays of elements of $M$, with boundary formula

$$
\begin{aligned}
\partial\left[\begin{array}{ll}
x & y \\
r & s
\end{array}\right]= & -[x, y]-[r, s]+[x+r, y+s] \\
& +[x, r]+[y, s]-[x+y, r+s] .
\end{aligned}
$$

The condition $\partial \partial=0$ is readily checked, using both the associative and commutative laws in $M$. In general, a $(q+1)$-cell of $Q(M)$ will be represented by an array of $2^{q}$ elements of $M$ at the $2^{q}$ vertices of a $q$-dimensional hypercube $C$. The boundary consists of $3 q$ cells, with suitable signs: the $2 q$ cells obtained from the $2 q$ faces of $C$, together with the $q$ cells formed by adding labels on corresponding vertices of opposite faces.

To state this formally, denote a vertex of the hypercube $C$ by a symbol $\left(\epsilon_{1}, \cdots, \epsilon_{q}\right)$ in which each $\epsilon_{i}$ is 0 or 1 . Then a cell $\sigma$ of dimension $q+1$ in $Q(M)$ is defined to be a function $\sigma$ with all symbols $\left(\epsilon_{1}, \cdots, \epsilon_{q}\right)$ as arguments and with values in $M$. The faces of $C$ are the $q$-cells $R_{i} \sigma$ and $S_{i} \sigma$ defined by

$$
\begin{aligned}
& \left(R_{i} \sigma\right)\left(\epsilon_{1}, \cdots, \epsilon_{q-1}\right)=\left(\epsilon_{1}, \cdots, \epsilon_{i-1}, 0, \epsilon_{i}, \cdots, \epsilon_{q-1}\right), \\
& \left(S_{i} \sigma\right)\left(\epsilon_{1}, \cdots, \epsilon_{q-1}\right)=\left(\epsilon_{1}, \cdots, \epsilon_{i-1}, 1, \epsilon_{i}, \cdots, \epsilon_{q-1}\right),
\end{aligned}
$$

for $i=1, \cdots, q$, and the cells formed by adding labels (in $M$ ) are

$$
\left(P_{i} \sigma\right)\left(\epsilon_{1}, \cdots, \epsilon_{q-1}\right)=\left(R_{i} \sigma\right)\left(\epsilon_{1}, \cdots, \epsilon_{q-1}\right)+\left(S_{i} \sigma\right)\left(\epsilon_{1}, \cdots, \epsilon_{q-1}\right) .
$$

The boundary of the $(q+1)$-cell $\sigma$ is then defined to be the $q$-chain

$$
\partial \sigma=\sum_{i=1}^{q}(-1)^{i+q}\left(R_{i} \sigma-S_{i} \sigma-P_{i} \sigma\right) .
$$


Here (but not in (12.5)) the addition is that of $q$-chains in $C_{q}(Q(M))$. This construction $Q$ has already been introduced, in connection with a topological problem, in $[3, \mathrm{I}]$; the boundary formula used here differs from that in [3] by the sign $(-1)^{q}$.

The proof that $\partial \partial \sigma=0$ follows formally from the rule

$$
V_{i} W_{j} \sigma=W_{i-1} V_{i} \sigma,
$$

where the letters $V$ and $W$ each stand for any one of the letters $P, R$, or $S$. The proof of this rule is straightforward, and the case $P_{i} P_{j}$ uses both the associative and commutative laws in $M$.

The construction $Q$ is employed with one of two appropriate normalizations. We define certain cells, called "slabs," by specifying the positions of zero entries, as follows. $A(q+1)$-cell $\sigma$ is an $i$-front slab $(i=1, \cdots, q)$ if $\epsilon_{i}=1$ implies $\sigma\left(\epsilon_{1}, \cdots, \epsilon_{q}\right)=0$, and an $i$-back slab if $\epsilon_{i}=0$ implies $\sigma\left(\epsilon_{1}, \cdots, \epsilon_{q}\right)=0$. In dimension 1 the zero cell [0] is also counted as a slab. The slabs of $Q(M)$ span a subcomplex $Q_{S}(M)$. Indeed, if $\sigma$ is an $i$-slab, and $V$ is one of the operators $R, S$, or $P$, then $V_{j} \sigma$ is an $(i-1)$-slab if $j<i$ and an $i$-slab if $j>i$, while for an $i$-front slab, $R_{i} \sigma=P_{i} \sigma$ and $S_{i} \sigma$ is the cell with all entries zero, and for an $i$-back slab $S_{i} \sigma=P_{i} \sigma$ and $R_{i} \sigma$ is the zero cell. Thus the boundary of an $i$-slab consists of $(i-1)$-slabs and $i$-slabs. The slab subcomplex $Q_{S}(M)$ contains the zero-normalization complex $Q_{0}(M)$. In low dimension, the slabs are cells of one of the forms

$$
[0] ;[x, 0],[0, y] ;\left[\begin{array}{ll}
x & y \\
0 & 0
\end{array}\right],\left[\begin{array}{ll}
0 & 0 \\
r & s
\end{array}\right],\left[\begin{array}{ll}
x & 0 \\
r & 0
\end{array}\right],\left[\begin{array}{ll}
0 & y \\
0 & s
\end{array}\right] .
$$

The second normalization complex $Q_{N}(M)$ is to consist of all slabs and all diagonals. The diagonals are determined by positions of zeros as follows: a $(q+1)$-cell $\sigma$ is an i-diagonal $(i=1, \cdots, q-1)$ if $\epsilon_{i} \neq \epsilon_{i+1}$ implies $\sigma\left(\epsilon_{1}, \cdots, \epsilon_{q}\right)=0$. The simplest diagonal cell is the 3-cell

$$
\left[\begin{array}{ll}
x & 0 \\
0 & s
\end{array}\right] \text {. }
$$

The diagonal cells span a subcomplex, for if $\sigma$ is an $i$-diagonal, and $V$ is one of $R, S$, or $P$, then $V_{j} \sigma$ is an (i-1)-diagonal if $j<i$, and an $i$-diagonal if $j>i+1$, while $V_{i} \sigma=V_{i+1} \sigma$. Hence $\partial \sigma$ consists of $(i-1)$ - and $i$-diagonals.

The construction $Q$ may be augmented by setting

$$
\partial^{\prime}[m]=\mu_{M}(m),
$$

just as in $\$ 1.0$. Each of the two normalizations $Q_{S}, Q_{N}$ is valid under this augmentation, since the only 1-cell in $Q_{S}$ or $Q_{N}$ is the cell [0].

THEOREM 12.1. The homology groups of the constructions $Q / Q_{N}$ and $Q / Q_{S}$ 
each satisfy the direct sum theorem

$$
H_{q}\left(M+M^{\prime}\right) \cong H_{q}(M)+H_{q}\left(M^{\prime}\right), \quad q=1,2, \cdots .
$$

Proof. Since the zero normalization is present, an argument with the direct sum diagrams (11.4), like that leading to $\left(11.7^{\prime}\right)$, reduces the proposition to the construction of a "splitting homotopy"

$$
E: Q(\lambda \rho)+Q\left(\lambda^{\prime} \rho^{\prime}\right) \simeq I: Q\left(M+M^{\prime}\right) \rightarrow Q\left(M+M^{\prime}\right)
$$

which maps the normalization complex into itself. For example, in low dimensions we must have

$$
\partial E\left[x+x^{\prime}\right]=[x]+\left[x^{\prime}\right]-\left[x+x^{\prime}\right],
$$

$\partial E\left[x+x^{\prime}, y+y^{\prime}\right]+E \partial\left[x+x^{\prime}, y+y^{\prime}\right]$

$$
=[x, y]+\left[x^{\prime}, y^{\prime}\right]-\left[x+x^{\prime}, y+y^{\prime}\right],
$$

where $x, y \in M, x^{\prime}, y^{\prime} \in M^{\prime}$, and $x+x^{\prime}=\left(x, x^{\prime}\right)$ in $M+M^{\prime}$.

The very nature of the construction $Q$ provides such a homotopy at once. In low dimensions one may set

$$
\begin{aligned}
E\left[x+x^{\prime}\right] & =\left[\begin{array}{ll}
x, & x^{\prime}
\end{array},\right. \\
E\left[x+x^{\prime}, y+y^{\prime}\right] & =\left[\begin{array}{ll}
x & x^{\prime} \\
y & y^{\prime}
\end{array}\right] .
\end{aligned}
$$

In other words, $E \sigma$ plasters the $M$ and $M^{\prime}$ contributions to $\sigma$ on opposite faces of a hypercube of one dimension higher. Explicitly, we define $E \sigma$ for a $(q+1)$-cell $\sigma$ of $Q\left(M+M^{\prime}\right)$ as

$$
\begin{aligned}
& (E \sigma)\left(\epsilon_{1}, \cdots, \epsilon_{q}, 0\right)=\lambda \rho\left(\sigma\left(\epsilon_{1}, \cdots, \epsilon_{q}\right)\right), \\
& (E \sigma)\left(\epsilon_{1}, \cdots, \epsilon_{q}, 1\right)=\lambda^{\prime} \rho^{\prime}\left(\sigma\left(\epsilon_{1}, \cdots, \epsilon_{q}\right)\right) .
\end{aligned}
$$

The proof that $E$ gives the homotopy follows formally from the readily established rules

$$
\begin{aligned}
& R_{i} E \sigma=E R_{i} \sigma, \quad S_{i} E \sigma=E S_{i} \sigma, \quad P_{i} E \sigma=E P_{i} \sigma, \quad i=1, \cdots, q, \\
& R_{q+1} E \sigma=Q(\lambda \rho) \sigma, \quad S_{q+1} E \sigma=Q\left(\lambda^{\prime} \rho^{\prime}\right) \sigma, \quad P_{q+1} E \sigma=\sigma .
\end{aligned}
$$

Furthermore $E$ respects (both) normalizations, mapping $Q_{N}\left(M+M^{\prime}\right)$ into $Q_{N}\left(M+M^{\prime}\right)$ and $Q_{S}\left(M+M^{\prime}\right)$ into $Q_{S}\left(M+M^{\prime}\right)$.

The splitting homotopy may also be used to establish generic acyclity.

THEOREM 12.2. The constructions $Q / Q_{N}$ and $Q / Q_{S}$ are generically acyclic for the category of associative and commutative systems, and are both generically and pseudo-generically acyclic for the category of abelian groups.

The second result shows that the pseudo-generic homology theory for abelian groups is identical with the generic homology theory; in other words 
the generic homology groups for an abelian group could be determined by a suitable construction in which inverses (but no repetitions) of letters are allowed in the boundary formulas.

The proof will be given in the case of $Q / Q_{S}$, for the category $\mathcal{X}_{A C}$; the other proofs are similar. Let $F=F_{A C}$ be the free system, and $z$ a generic $(q+1)$ dimensional cycle in the augmented complex $\bar{Q}\left(F, \Phi^{*}\right)$ modulo $\bar{Q}_{S}\left(F, \Phi^{*}\right)$. Let $g_{n}$ be the last generator of $F$ which actually occurs in any one of the cells of the cycle $z$. We show by induction on $n$ that $z$ is a boundary in $\bar{Q}\left(F, \Phi^{*}\right) / \bar{Q}_{N}\left(F, \Phi^{*}\right)$. The proof for $n=0$ or $n=1, q=-1$, is trivial. If $n=1$ and $q=0, z$ is a multiple of $\left[g_{1}\right]$, hence is not a cycle unless it is identically zero. If $n=1$ and $q>0$, each cell occurring in $z$ has at most one nonzero entry, and hence is necessarily a slab. If $n>1$, let $M$ denote the free associative and commutative system on the generators $g_{1}, \cdots, g_{n-1}$, and $M^{\prime}$ the free system on the remaining generators. Then $F=M+M^{\prime}$ and $Q\left(F, \Phi^{*}\right)$ $C Q\left(M+M^{\prime}\right)$. The splitting homotopy $E$ applied to the cycle $z$ yields.

$$
z \equiv Q(\lambda \rho) z+Q\left(\lambda^{\prime} \rho^{\prime}\right) z-\partial E z\left(\bmod Q_{S}\left(M+M^{\prime}\right)\right) .
$$

The construction of $E$ shows that $E z$ is still a generic chain. Furthermore $Q(\lambda \rho) z$ and $Q\left(\lambda^{\prime} \rho^{\prime}\right) z$ are generic cycles involving at most $n-1$ and 1 generators respectively, hence they are boundaries by the induction assumption. The proof is complete.

The use of a normalization is essential to this proof (for example, in the treatment of the case $n=1$ above). Indeed, the construction $Q$ with no normalization is not generically acyclic on the category $\mathcal{X}_{A C}$, because $\left[g_{1}, 0\right]$ $-[0,0]$ is a generic 2-cycle, but is not the boundary of a generic chain (because the boundary of any 3 -cell $\sigma$ in which $g_{1}$ is the only nonzero entry is the zero cell). This example might suggest the use of a different "single entry" normalization, containing all cells with at most one nonzero entry. However, for this normalization, the cell

$$
\left[\begin{array}{ll}
g_{1} & 0 \\
g_{2} & 0
\end{array}\right]
$$

is a generic 3-cycle, but not a boundary.

13. Successive elimination of generators. Generic acyclicity may of ten be established by successively eliminating the generators in a proposed generic cycle. Formally, it suffices to eliminate the first generator; this process may be described, for a free system $F$ with generators $g_{1}, g_{2}, \cdots$, in terms of the generic endomorphism $\nu: F \rightarrow F$ with

$$
\nu\left(g_{1}\right)=1, \quad \nu\left(g_{i}\right)=g_{i},
$$

THEOREM 13.1. Let $\bar{K}$ be an augmented construction on the category $\mathcal{H}(F)$, I the identity map of the generic subcomplex $\bar{K}\left(F, \Phi^{*}\right)$, and $\bar{K}(\nu)$ the map in- 
duced by (13.1). If there is a homotopy

$$
S: \bar{K}(\nu) \simeq I: \bar{K}\left(F, \Phi^{*}\right) \rightarrow \bar{K}\left(F, \Phi^{*}\right),
$$

which carries every cell with all entries 1 into the zero chain, then $\bar{K}$ is generically acyclic modulo the zero normalization.

Proof. Consider the generic endomorphisms $\lambda, \mu, \nu_{n}: F \rightarrow F$ with

$$
\begin{aligned}
& \lambda\left(g_{1}\right)=1, \quad \lambda\left(g_{i+1}\right)=g_{i}, \\
& i=1,2, \cdots, \\
& \mu\left(g_{i}\right)=g_{i+1}, \\
& i=1,2, \cdots \text {, } \\
& \nu_{n}\left(g_{i}\right)=1, \quad i \leqq n, \quad \nu_{n}\left(g_{i}\right)=g_{i}, \\
& i>n \text {. }
\end{aligned}
$$

Here $\nu_{0}=I, \nu_{1}=\nu$, and $\mu \nu_{n} \lambda=\nu_{n+1}$. We are given $S$ with

$$
\partial S+S \partial=I-\bar{K}(\nu) ;
$$

define $S_{1}=S$ and $S_{n+1}=\bar{K}\left(\mu^{n}\right) S \bar{K}\left(\lambda^{n}\right)$. Then

$$
\begin{aligned}
\partial S_{n+1}+S_{n+1} \partial & =\bar{K}\left(\mu^{n} \lambda^{n}\right)-\bar{K}\left(\mu^{n} \nu \lambda^{n}\right) \\
& =\bar{K}\left(\nu_{n}\right)-\bar{K}\left(\nu_{n+1}\right) .
\end{aligned}
$$

We set $T=S_{1}+S_{2}+\cdots$. Any cell $\sigma$ of $\bar{K}\left(F, \Phi^{*}\right)$ involves at most a finite number of generators $g_{1}, \cdots, g_{n}$. Hence $\bar{K}\left(\lambda^{n}\right) \sigma$ is the cell with all entries $1, S \bar{K}\left(\lambda^{n}\right) \sigma$ is zero, and the formally infinite sum $T \sigma$ is actually finite. Furthermore $\bar{K}\left(\nu_{n}\right) \sigma=\sigma_{0}$ is the cell of the same type as $\sigma$, with all entries 1 . Therefore,

$$
\partial T \sigma+T \partial \sigma=\sigma-\sigma_{0}
$$

or

$$
\partial T+T \partial \equiv I\left(\bmod \bar{K}_{0}\left(F, \Phi^{*}\right)\right) .
$$

This homotopy asserts that $\bar{K}\left(F, \Phi^{*}\right)$ is acyclic modulo the zero normalization $\bar{K}_{0}\left(F, \Phi^{*}\right)$.

We illustrate this result by proving the generic acyclicity in dimensions 1,2 , and 3 of the construction $A$ described in the introduction for the category $\mathscr{X}_{A C}$ or $\mathfrak{X}_{G A}$. This construction is augmented by defining

$$
\partial^{\prime}: C_{1}(A(M)) \rightarrow H(M)
$$

as $\partial^{\prime}[x]=\mu_{M}(x)$; by Lemma 8.1 the group $H(F)=H\left(F, \Phi^{*}\right)$ for $F=F_{A C}$ or $F=F_{G A}$ is just the free abelian group on the generators $g_{i}=\mu_{F}\left(g_{i}\right)$, $i=1,2, \cdots$.

To define $S$, it is convenient to denote the first generator $g_{1}$ by $h$ and to denote generic elements of $F$ not involving $h$ by $x, y, \cdots$ Then any generic element of $F$ has one of the forms $x$ or $x+h$ (additive notation), and $h$ appears at most once in each generic cell. For an element $u=m g_{1}^{\prime}+v$ of $H(F)$, 
where $v$ does not involve the generator $g_{1}^{\prime}$, we set $S u=m[h]$. Then $\partial^{\prime} S u$ $=m g_{1}^{\prime}=u-\bar{K}(\nu) u$, as desired. Any generic cell of dimension 1 has one of the forms $[x]$ or $[x+h]$; we set

$$
\begin{aligned}
S[x+h] & =-[x, h], \\
S[x] & =0,
\end{aligned}
$$

and verify (13.5). Similarly, in dimensions 2 and 3 we set

$$
\begin{aligned}
S[x, y] & =0, \quad S[x, y+h]=[x, y, h], \\
S[x+h, y] & =-[x, h, y]+[x, y, h]+[h \mid y] \\
S[x, y, z] & =0, \quad S[x, y, z+h]=-[x, y, z, h], \\
S[x, y+h, z] & =[x, y, h, z]-[x, y, z, h], \\
S[x+h, y, z] & =[x, h, y, z]-[x, y, h, z]+[x, y, z, h]+[h \mid y, z], \\
S[x \mid y+h] & =-[x \mid y, h]-[x \| h], \\
S[x+h \mid y] & =-[x, h \mid y], \quad S[x \mid y]=0 .
\end{aligned}
$$

The condition (13.5) is again readily verified in each case. Hence $\bar{A}$ is generically acyclic, modulo the zero normalization $\bar{A}_{0}$, in dimensions $0,1,2$, and 3. One may also show that $\bar{A}_{0}\left(F, \Phi^{*}\right)$ is acyclic, hence that $\bar{A}$ is generically acyclic, without any normalization.

The same proof can be carried out for all dimensions of the construction $A$, using the general description of this construction, as given in [3, II]. Given the cells of $A$ through dimension $q$, this method of successive elimination can be used to find the cells to be added in dimension $q+1$ to the cells already present in $A^{0}$, and thus provides a refinement of the existence proof for generically acyclic constructions.

The method of successive eliminations can also be used to prove that the cubical constructions $Q / Q_{N}$ and $Q / Q_{S}$ are generically cyclic for the category $\mathcal{X}_{A C}$. Furthermore, the generic acyclicity of $Q / Q_{N}$ and $A / A_{0}$ as proved above shows that in dimensions 1,2 , and 3 , these two constructions yield the same homology and cohomology groups for any associative and commutative system $M$.

We do not have an explicit freely acyclic construction for the categories $\mathcal{X}_{A C}$ and $\mathcal{X}_{G A}$, although the existence theorem demonstrates that there must be such constructions. However, we can prove that the homology theory obtained by a freely acyclic construction is different from the generically acyclic homology theory (and hence that the constructions $Q / Q_{N}, Q / Q_{S}$, and $A$ are not freely acyclic in $\mathcal{X}_{A C}$ ). For let $F=F_{A C}$, choose any generator $g$ of $F$, and construct a bilinear function $b$ on $F \times F$ to a suitable coefficient group $G$ in such fashion that $b(g, g) \neq 0$. Using this bilinear function we can define a 3dimensional cochain $f$ of $C^{3}(A(F), G)$ as 


$$
\begin{aligned}
f([x \mid y]) & =b(x, y), \\
f([x, y, z]) & =0 .
\end{aligned}
$$

Then $\delta f=0$, and $f$ is a cocycle. However $f([g \mid g]) \neq 0$, hence the 3 -chain $[g \mid g]$ of $A(F)$ is not a boundary. However $\partial[g \mid g]=0$. Hence $[g \mid g]$ is a nontrivial three-dimensional cycle of the complex $A(F)$, and $H_{3}(A(F)) \neq 0$. Thus the construction $A$ cannot give the freely acyclic homology groups, for which $H_{3}(F)=0$.

For an abelian group $M$, and any coefficient group $G$, there are two generically acyclic cohomology theories, obtained by regarding $M$ as an element of $\mathcal{X}_{A}$ or as an element of $\mathfrak{X}_{A C}$. In the first case, $H^{2}(M, G)$ is the well known group of central group extensions of $G$ by $M$ [cf. 1]. In the second case, inspection of the construction $A$ shows that $H^{2}(M, G)$ is the group of abelian group extensions of $G$ by $M$.

14. Classification of generic relations. We return to the concepts and notations of $\S 2$. As in $\S 8$ we call an identity $\left(w_{1}, w_{2}\right)$ generic if $w_{1}$ and $w_{2}$ are generic elements of $F_{I}$ which involve the same letters $g_{i}$. A relation $R$ is called generic if it consists of generic identities only.

TheOREM 14.1. Any generic relation $R$ has the same closure as one and only one of the relations $I, A, C$, or $A C$.

Proof. If $R$ consists only of the trivial identities $(w, w)$, then clearly $\bar{R}=\bar{I}$. Let $\left(w_{1}, w_{2}\right)$ be a nontrivial generic identity in $\bar{R}$. We shall say that $\left(w_{1}, w_{2}\right)$ is order preserving if the generators in $w_{1}$ occur in the same order as in $w_{2}$. Otherwise $\left(w_{1}, w_{2}\right)$ is said to be order reversing.

We first show that if $\bar{R}$ contains a nontrivial order preserving generic identity, then $A \subset \bar{R}$. Indeed let $\left(w_{1}, w_{2}\right)$ be a nontrivial order preserving generic identity in $\bar{R}$ involving the least possible number of generators. Let $w_{1}=u_{1} v_{1}, w_{2}=u_{2} v_{2}$. If $u_{1}$ and $u_{2}$ involve the same generators, then choosing $\alpha: F \rightarrow F$ so that $\alpha u_{1}=u, \alpha u_{2}=u_{2}, \alpha v_{1}=1, \alpha v_{2}=1$, we arrive at $\left(u_{1}, u_{2}\right) \in R$, so that $u_{1}=u_{2}$. Similarly $v_{1}=v_{2}$, and therefore $\left(w_{1}, w_{2}\right)$ is trivial. Thus we may assume that there exist three generators, say $g_{1}, g_{2}, g_{3}$, appearing in $w_{1}$ and in $w_{2}$ in that order, which are such that $g_{1}$ and $g_{2}$ are in $u_{1}, g_{3}$ is in $v_{1}$, while $g_{1}$ is in $u_{2}$ and $g_{2}$ and $g_{3}$ are in $v_{2}$. Choosing $\alpha: F \rightarrow F$ so that $g_{1}, g_{2}, g_{3}$ are mapped into themselves while all other generators are mapped into 1 , we find that $\alpha w_{1}=\left(g_{1} g_{2}\right) g_{3}$ and $\alpha w_{2}=g_{1}\left(g_{2} g_{3}\right)$. Thus $A \subset \bar{R}$.

An even easier argument shows that if $\bar{R}$ contains an order reversing generic identity, then $C \subset \bar{R}$.

Suppose now that $\bar{R}$ contains nontrivial generic identities and that they are all order preserving. Since every order preserving generic identity is in $\bar{A}$, it follows from $A \subset \bar{R}$ that $\bar{R}=\bar{A}$.

Suppose now that $\bar{R}$ contains nontrivial generic identities, but that they 
are all order reversing. We shall show that $\bar{R} \subset \bar{C}$, thus proving $\bar{R}=\bar{C}$. Suppose instead that $\bar{R} \subset \bar{C}$ fails, and let $\left(w_{1}, w_{2}\right)$ be a generic identity in $\bar{R}$ but not in $\bar{C}$, involving the least possible number of generators. Let $w_{1}=u_{1} v_{1}$, $w_{2}=u_{2} v_{2}$. If $u_{1}$ and $u_{2}$ involve the same generators, then, as before, $\left(u_{1}, u_{2}\right)$ and $\left(v_{1}, v_{2}\right)$ are in $\bar{R}$. Thus $\left(u_{1}, u_{2}\right)$ and $\left(v_{1}, v_{2}\right) \in \bar{C}$ imply $\left(w_{1}, w_{2}\right) \in \bar{C}$. If $u_{1}$ and $v_{2}$ involve the same generators, then again by suitable maps $\alpha: F \rightarrow F$, we show that $\left(u_{1}, v_{2}\right)$ and $\left(v_{1}, u_{2}\right)$ are in $\bar{R}$. Thus $\left(u_{1}, v_{2}\right) \in \bar{C}$ and $\left(v_{1}, u_{2}\right) \in \bar{C}$. Consequently $\left(u_{1} v_{1}, v_{2} u_{2}\right) \in \bar{C}$. Since $\left(v_{2} u_{2}, u_{2} v_{2}\right) \in \bar{C}$, it follows that $\left(w_{1}, w_{2}\right) \in \bar{C}$. Hence we may assume that $u_{1}$ involves two generators $g_{1}$ and $g_{2}$, of which $g_{1}$ occurs in $u_{2}$ while $g_{2}$ occurs in $v_{2}$. Let $g_{3}$ be any generator occurring in $v_{1}$. Choose $\alpha: F \rightarrow F$, so that $g_{1}, g_{2}, g_{3}$ are mapped into themselves while all other generators are mapped into 1 . Then $\alpha w_{1}$ will be either $\left(g_{1} g_{2}\right) g_{3}$ or $\left(g_{2} g_{1}\right) g_{3}$, while $\alpha w_{2}$ will be either $\left(g_{3} g_{1}\right) g_{2}$ or $\left(g_{1} g_{3}\right) g_{2}$, or $g_{1}\left(g_{3} g_{2}\right)$ or $g_{1}\left(g_{2} g_{3}\right)$ depending upon the position of $g_{3}$ in $w_{2}$. In either case, using the fact that $C \subset \bar{R}$, we find that the associative law is in $\bar{R}$, contrary to the assumption that all generic relations in $\bar{R}$ are order reversing.

There remains the case when $R$ contains nontrivial identities of both the order preserving and the order reversing type. Then $A \subset \bar{R}$ and $C \subset \bar{R}$, so that $A C \subset \bar{R}$. However $\bar{A} \bar{C}$ contains all generic identities. Therefore

$$
\bar{R}=\bar{A} \bar{C} \text {. }
$$

15. Systems without a unit. The previous considerations assumed that all systems $M \in \mathcal{X}(F)$ had a unit element. It is possible to build a similar theory without this assumption. We shall give here a brief outline of the modifications which have to be made and of the peculiarities which arise.

Let $F_{I}$ be the universal free system (with unit) generated by $g_{1}, g_{2}, \cdots$, $g_{k}, \cdots$ and denote by $G_{I}$ the system $F_{I}$ with the unit element removed. For any relation $R$ we obtain by factorization of $G_{I}$, as in the proof of Theorem 2.1, a free system $G_{R}$ and a corresponding category $\mathcal{N}_{R}$ consisting of all systems satisfying the identities of $R$. Note that $\mathcal{N}_{R}$ contains $\mathcal{X}_{R}$ as a subcategory, but that $G_{R}$ need not be a subsystem of $F_{R}$. Since every element of $G_{R}$ is an actual product of generators, we may consider the subsystem $G_{R}^{n}$ generated by $g_{1}, \cdots, g_{n}$.

In place of the admissible class $\Phi$ of endomorphisms $\phi: F_{R} \rightarrow F_{R}$, we shall consider here a class $\Psi$ of homomorphisms $\alpha: G_{R}^{n} \rightarrow G_{R}^{m}$ for any $n, m$. We shall limit ourselves to two such classes $\Psi$, namely the class $\Psi^{0}$ of all such homomorphisms and the class $\Psi^{*}$ of generic homomorphisms. Thus in effect we limit ourselves to the theories of free and generic acyclicity only and to the categories $N_{R}$ defined by sets of relations.

The definition of a construction and of an augmented construction on $\mathcal{N}_{R}$ remains unchanged. The typical cell $\tau=\left[g_{1}, \cdots, g_{r}\right]_{t}$ of type $t$ will be defined as a cell of $K\left(G_{R}^{r}, \Psi\right)$, and in the canonical representation $\sigma=K(\alpha) \tau$ of a cell $\sigma \in K(N)$ of type $t$ we shall have $\alpha: G_{R}^{r} \rightarrow N$. 
The first significant difference occurs in the definition of $\Psi$-acyclicity in §6. Instead of considering the complex $K\left(F_{R}\right)$ we consider the sequence of complexes $K\left(G_{R}^{n}\right)(n=1,2, \cdots)$ and formulate the analogues of (6.1) for each complex of this sequence. The proof of the analogues of the results of $\$ 6$ carry over with minor changes only.

The considerations concerning normalizations ( $\$ \S 8-9)$ do not apply since units are not universally present. The classification theorem of $\$ 14$ is false; the set $R$ consisting of the identity $g_{1}\left(g_{2} g_{3}\right)=\left(g_{2} g_{1}\right) g_{3}$ does not have the same closure as any of the sets $I, A, C$, or $A C$ (in the case with units this set $R$ has the same closure as $A C$ ).

We shall now discuss the question of $\Psi$-acyclic constructions on the categories $\mathcal{N}_{R}$, where $R$ is one of $I, A, C$, or $A C$. In these cases it is easy to see that $G_{R}$ is obtained from $F_{R}$ by removing the unit element.

An augmented $\Phi$-construction $\bar{K}$ on the category $\mathcal{X}_{R}(R=I, A, C$, or $A C)$ will be called regular, if:

(15.1) The full normalization $K_{1}$ or $\bar{K}$ is a normalization (that is, $K_{1}(M)$ is a subcomplex of $\bar{K}(M)$ ).

(15.2) $\bar{K}$ is $\Phi$-acyclic $\bmod K_{1}$.

(15.3) The complex $\bar{K}\left(F_{R}, \Phi\right)$ is the disjoint union of its subcomplex $\bar{K}_{1}\left(F_{R}, \Phi\right)=K_{1}\left(F_{R}\right) \cap \bar{K}\left(F_{R}, \Phi\right)$ and a complex $\bar{K}^{\prime}\left(F_{R}, \Phi\right)$.

This last property means that the boundary of any $\Phi$-cell which has no entries 1 is a chain involving only such cells. Thus the boundary of a typical cell yields a boundary formula which can be used to extend the construction to the category $\mathcal{N}_{R}$. It can be proved that the resulting construction is $\Psi$ acyclic.

In the case $R=I$ the construction $\bar{K}$ of $\S 10$ is regular and thus yields a generically and freely acyclic construction on $\mathcal{N}_{I}$. A system $M$ with a unit may be regarded either as an element of $\mathcal{N}_{I}$ or of $\mathcal{X}_{I}$. In the first case the groups of $M$ are those of the complex $K(M)$ while in the second they are those of the complex $K(M) / K_{1}(M)$.

The same applies to the case $R=C$ if we use the constructions $K$ and $L$ as described in $\S 10$.

In the case $R=A$, the construction $A^{0}$ of $\S 7$ is regular and therefore yields a generically and freely acyclic construction for $\mathcal{N}_{A}$. If $M \in \mathcal{X}_{A}$, then the groups of $M$ are the same whether $M$ is regarded as an element of $\mathcal{X}_{A}$ or of $\mathcal{N}_{A}$. This is due to the fact that the groups of $A^{0}(M)$ and $A^{0}(M) / A_{1}^{0}(M)$ are naturally isomorphic.

The cubical construction $Q$ on $\mathscr{X}_{A C}$ as described in $\$ 12$ is not regular since it does not admit the full normalization. The construction $A$, however, the beginning of which appears in the introduction and in $\$ 13$, is regular and thus yields an example of a generically acyclic construction on the category $N_{A C}$. A full exposition of the construction $A$ for commutative and associative systems will appear elsewhere. 


\section{BIBLIOGRAPHY}

1. Samuel Eilenberg, Topological methods in abstract algebra, cohomology theory of groups, Bull. Amer. Math. Soc. vol. 55 (1949) pp. 3-37.

2. Samuel Eilenberg and Saunders MacLane, General theory of natural equivalences, Trans. Amer. Math. Soc. vol. 58 (1945) pp. 231-294.

3. - Cohomology theory of abelian groups and homotopy theory. I and II, Proc. Nat. Acad. Sci. U.S.A. vol. 36 (1950) pp. 443-447 and 657-663.

4. Roger Lyndon, New proof for a theorem of Eilenberg and MacLane, Ann. of Math. vol. 50 (1949) pp. 731-735.

Columbia University,

NEW YORK, N. Y.

The University of Chicago,

Chicago, Ill. 